\title{
Octant Degeneracy and Quadrant of Leptonic CPV Phase at Long Baseline $v$ Experiments and Baryogenesis
}

\author{
Kalpana Bora, ${ }^{1}$ Gayatri Ghosh, ${ }^{1}$ and Debajyoti Dutta ${ }^{2}$ \\ ${ }^{1}$ Department of Physics, Gauhati University, Guwahati 781014, India \\ ${ }^{2}$ Harish-Chandra Research Institute, Chhatnag Road, Jhunsi, Allahabad 211019, India \\ Correspondence should be addressed to Kalpana Bora; kalpana.bora@gmail.com
}

Received 30 May 2016; Revised 22 July 2016; Accepted 8 August 2016

Academic Editor: Abhijit Samanta

Copyright (c) 2016 Kalpana Bora et al. This is an open access article distributed under the Creative Commons Attribution License, which permits unrestricted use, distribution, and reproduction in any medium, provided the original work is properly cited. The publication of this article was funded by SCOAP ${ }^{3}$.

\begin{abstract}
In a recent work by us, we have studied how $\mathrm{CP}$ violation discovery potential can be improved at long baseline neutrino experiments (LBNE/DUNE), by combining with its ND (near detector) and reactor experiments. In this work, we discuss how this study can be further analysed to resolve entanglement of the quadrant of leptonic CPV phase and octant of atmospheric mixing angle $\theta_{23}$, at LBNEs. The study is done for both NH (normal hierarchy) and IH (inverted hierarchy), HO (higher octant), and LO (lower octant). We show how baryogenesis can enhance the effect of resolving this entanglement and how possible values of the leptonic $\mathrm{CP}$ violating phase $\delta_{\mathrm{CP}}$ can be predicted in this context. With respect to the latest global fit data of neutrino mixing angles, we predict the values of $\delta_{\mathrm{CP}}$ for different cases. In this context we present favoured values of $\delta_{\mathrm{CP}}\left(\delta_{\mathrm{CP}}\right.$ range at $\left.\geq 2 \sigma\right)$ constrained by the latest updated BAU range and also confront our predictions of $\delta_{\mathrm{CP}}$ with an up-to-date global analysis of neutrino oscillation data. We find that some region of the favoured $\delta_{\mathrm{CP}}$ parameter space lies within the best fit values around $\delta_{\mathrm{CP}} \simeq 1.3 \pi-1.4 \pi$. A detailed analytic and numerical study of baryogenesis through leptogenesis is performed in this framework within the nonsupersymmetric $\mathrm{SO}(10)$ models.
\end{abstract}

\section{Introduction}

Today, physics is going through precision era; this is more so for neutrino physics. With the measurement of reactor mixing angle $\theta_{13}[1-7]$ precisely by reactor experiments, the unknown quantities left to be measured in neutrino sector are leptonic $\mathrm{CP}$ violating phase [8-13], octant of atmospheric angle $\theta_{23}[14-20]$, mass hierarchy, nature of neutrino, and so forth. Long baseline neutrino experiments (LBNE [21, 22], NO $v$ A [23], T2K [24], MINOS [25], LBNO [26], etc.) may be very promising, in measuring many of these sensitive parameters.

Measuring leptonic $\mathrm{CP}$ violation (CPV) is one of the most demanding tasks in future neutrino experiments [27]. The relatively large value of the reactor mixing angle $\theta_{13}$ measured with a high precision in neutrino experiments [15] has opened up a wide range of possibilities to examine $\mathrm{CP}$ violation in the lepton sector. The leptonic $\mathrm{CPV}$ phase can be induced by the PMNS neutrino mixing matrix [28-30] which holds, in addition to the three mixing angles, a Dirac-type CP violating phase in general as it exists in the quark sector and two extra phases if neutrinos are Majorana particles. Even if we do not yet have significant evidence for leptonic CPV, the current global fit to available neutrino data manifests nontrivial values of the Dirac-type CP phase [31-34]. In this context, possible size of leptonic CP violation detectable through neutrino oscillations can be predicted. Recently, [8], we have explored possibilities of improving CP violation discovery potential of newly planned long baseline neutrino experiments (earlier LBNE, now called DUNE) in USA. In neutrino oscillation probability expression $P\left(v_{\mu} \rightarrow v_{e}\right)$ relevant for LBNEs, the term due to significant matter effect changes sign when oscillation is changed from neutrino to antineutrino mode, or vice versa. Therefore in the presence of matter effects, CPV effect is entangled and hence one has two degenerate solutions, one due to CPV phase and another due to its entangled value. It has been suggested to resolve this issue by combining two experiments with different 
baselines $[35,36]$. But CPV phase measurement depends on value of reactor angle $\theta_{13}$, and hence precise measurement of $\theta_{13}$ plays crucial role in its CPV measurements. This fact was utilized recently by us [8], where we have explored different possibilities of improving CPV sensitivity for LBNE (USA). We did so by considering LBNE with

(1) its ND (near detector),

(2) reactor experiments.

We considered both appearance $\left(\nu_{\mu} \rightarrow \nu_{e}\right)$ and disappearance $\left(\nu_{\mu} \rightarrow \nu_{e}\right)$ channels in both neutrino and antineutrino modes. Some of the observations made in [8] are as follows:

(1) CPV discovery potential of LBNE increases significantly when combined with near detector and reactor experiments.

(2) CPV violation sensitivity is more in LO (lower octant) of atmospheric angle $\theta_{23}$, for any assumed true hierarchy.

(3) CPV sensitivity increases with mass of FD (far detector).

(4) When $\mathrm{NH}$ is true hierarchy, adding data from reactors to LBNE improves its CPV sensitivity irrespective of octant.

Aim of this work is to critically analyse the results presented in [8], in context of entanglement of quadrant of CPV phase and octant of $\theta_{23}$, and hence study the role of baryogenesis in resolving this entanglement. Though in [8] we studied effect of both ND and reactor experiments on CPV sensitivity of the LBNEs, in this work we have considered only the effect of ND. But similar studies can also be done for the effect of reactor experiments on LBNEs as well. The details of LBNE and ND are same as in [8]. Following the results of [8], either of the two octants is favoured, and the enhancement of CPV sensitivity with respect to its quadrant is utilized here to calculate the values of lepton-antilepton symmetry. This is done considering two cases of the rotation matrix for the fermions, CKM only and CKM + PMNS. Then, this is used to calculate the value of BAU within the nonsupersymmetric $\mathrm{SO}(10)$ model [37], characterized by the presence of an intermediate mass scale where both the lepton number conservation and quark-lepton symmetry are broken. In the supersymmetric case, the two effects occur at the unification scale, and no intermediate scale is present.

This is an era of precision measurements in neutrino physics. We therefore consider variation of $\Delta m_{31}^{2}$ range at its $3 \sigma \mathrm{CL}$ versus $\delta_{\mathrm{CP}}$ range at $\geq 2 \sigma$ over the corresponding distribution of $\chi^{2}$-minima from Figure 2. We calculate baryon to photon ratio and compare with its experimentally known best fit value. As constrained by the latest updated BAU limits, $5.7 \times 10^{-10}<$ BAU $<6.7 \times 10^{-10}$, we plot $\theta_{13}$ range at its $3 \sigma \mathrm{CL}[6]$ from its central value versus $\delta_{\mathrm{CP}}$ range at $\geq 2 \sigma$ over $\chi^{2}$-minima distribution and find that for $\mathrm{IH}$ and LO case the allowed $\theta_{13}$ has a varied range altering within $8.7^{\circ}$ to $9.4^{\circ}$ in the upper quadrant ( $\pi$ to $2 \pi$ ) and $8.7^{\circ}$ to $9.8^{\circ}$ in the lower quadrant ( 0 to $\pi$ ). Similarly to $\mathrm{IH}$ and $\mathrm{HO}$ case the allowed $\theta_{13}$ has a varied range differing within $8.7^{\circ}$ to $9.8^{\circ}$. As shown in our results in Section 4 , in IH and LO case the spectrum of $\delta_{\mathrm{CP}}$ is mostly concentrated in the region $88^{\circ}$ for $\theta_{13}$ around $9.09^{\circ}$ to $9.2^{\circ}, 9.25^{\circ}, 9.35^{\circ}$ to $9.5^{\circ}$, and $9.6^{\circ}$ to $9.65^{\circ}$. Also $\delta_{\mathrm{CP}}=276.5^{\circ}$ exists for $\theta_{13}$ around $9.06^{\circ}$ to $9.12^{\circ}, 9.3^{\circ}$ to $9.45^{\circ}, 9.517^{\circ} . \delta_{\mathrm{CP}}=290^{\circ}$ survives for $\theta_{13}$ around, $9^{\circ}, 9.15^{\circ}$ to $9.3^{\circ}, 9.35^{\circ}$ to $9.5^{\circ}$ in the higher quadrant ( $\pi$ to $2 \pi$ ). Similarly as shown in our results in Section 4 , as allowed by the updated BAU limits in $\mathrm{IH}$ and $\mathrm{HO}$ case, the parameter space of $\delta_{\mathrm{CP}}$ in the lower quadrant $\left(0\right.$ to $\pi$ ) demands $\delta_{\mathrm{CP}}$ to be around $69^{\circ}$, for $\theta_{13} \sim 8.86^{\circ}-8.896^{\circ}, 9.06^{\circ}-9.22^{\circ}, 9.32^{\circ}$, and $9.61^{\circ}$. There also exists $\delta_{\mathrm{CP}}=95^{\circ}$ which constrains $\theta_{13}$ to be around $8.99^{\circ}$, $9.24^{\circ}, 9.352^{\circ}, 9.5-9.64^{\circ}$, and $9.79^{\circ}$. In the upper quadrant $(\pi$ to $2 \pi$ ) for the present updated BAU constraint, the allowed region of $\delta_{\mathrm{CP}}$ parameter space becomes constrained with $\delta_{\mathrm{CP}}=257.5^{\circ}$, for $\theta_{13}$ around $8.96^{\circ}, 9.0974^{\circ}$ to $9.22^{\circ}, 9.5^{\circ}, 9.6^{\circ}$, and $9.74^{\circ}$. Also the BAU constraint requires $\delta_{\mathrm{CP}}$ to be equal to $288^{\circ}$, for $\theta_{13}$ around $9^{\circ}, 9.06^{\circ}$ to $9.35^{\circ}, 9.5^{\circ}, 9.69^{\circ}$, and $9.8^{\circ}$. Also $\delta_{\mathrm{CP}}=295^{\circ}$ survives for $\theta_{13}$ around $9.09^{\circ}, 9.26^{\circ}, 9.34^{\circ}$, $9.5^{\circ}, 9.6^{\circ}$, and $9.69^{\circ}$. A part of the allowed $\delta_{\mathrm{CP}}$ parameter space is found to lie within the best fit values of $\delta_{\mathrm{CP}} \simeq 1.3 \pi-1.4 \pi$. As constrained by the current BAU bounds we present the $3 \mathrm{D}$ variation of the favoured range of parameters: $\Delta m_{31}^{2}$ range within its $3 \sigma$ C.L, $\theta_{13}$ range at its $3 \sigma \mathrm{CL}$, and $\delta_{\mathrm{CP}}$ range at $\geq 2 \sigma$ varied within $[0,2 \pi]$ in Figure 2.

As can be seen from the results presented in Section 4, from Figures 3(a) and 3(b), we find that BAU can be explained most favourably for the following possible cases: $\delta_{\mathrm{CP}}=$ $1.536 \pi, \mathrm{IH}$ and LO of $\theta_{23} ; \delta_{\mathrm{CP}}=1.611 \pi$, IH and LO of $\theta_{23}$; $\delta_{\mathrm{CP}}=0.488 \pi$, IH and LO of $\theta_{23} ; \delta_{\mathrm{CP}}=1.638 \pi$, IH and $\mathrm{HO}$ of $\theta_{23} ; \delta_{\mathrm{CP}}=1.6 \pi, \mathrm{IH}$ and $\mathrm{HO}$ of $\theta_{23} ; \delta_{\mathrm{CP}}=1.43 \pi, \mathrm{IH}$ and $\mathrm{HO}$ of $\theta_{23} ; \delta_{\mathrm{CP}}=0.777 \pi$, IH and $\mathrm{HO}$ of $\theta_{23} ; \delta_{\mathrm{CP}}=0.5277 \pi, \mathrm{IH}$ and $\mathrm{HO}$ of $\theta_{23} ; \delta_{\mathrm{CP}}=1.436 \pi, \mathrm{NH}$ and $\mathrm{HO}$ of $\theta_{23}$. It is worth mentioning that the value of $\delta_{\mathrm{CP}}=1.43 \pi$ and $\delta_{\mathrm{CP}}=1.436 \pi$ is close to the central value of $\delta_{\mathrm{CP}}$ from the recent global fit result $[31,34,38-42]$. It is fascinating to notice that a nearly maximal CP-violating phase $\delta_{\mathrm{CP}}=(3 / 2) \pi$ has been reported by the T2K [43], NO $v \mathrm{~A}$ [44], and Super-Kamiokande experiments [45], even if the statistical significance of all these experimental results is below $3 \sigma$ level. This accords with one of our calculated favoured solutions $\delta_{\mathrm{CP}}=1.536 \pi$ which exactly holds with the current BAU constraints. Moreover, such hints of a nonzero $\delta_{\mathrm{CP}}$ were already present in global analyses of neutrino oscillation data, such as the one in [3133]. Our main aim in this work is to carry out a detailed analysis of the breaking of the entanglement of the quadrant of leptonic CPV phase and octant of $\theta_{23}$ by using the current data of $\nu$ mixing parameters and identify the CPV phase and $\theta_{13}$ spectrum required to get the breaking favourable with the current BAU constraint. These results could be important keeping in view that the quadrant of leptonic CPV phase and octant of atmospheric mixing angle $\theta_{23}$ are yet not fixed. Also, they are significant in context of precision measurements on neutrino oscillation parameters.

The paper is organized as follows. In Section 2, we discuss entanglement of quadrant of CPV phase and octant of $\theta_{23}$. In Section 3, we present a review on leptogenesis and baryogenesis. In Section 4 we show how the baryon asymmetry (BAU) 


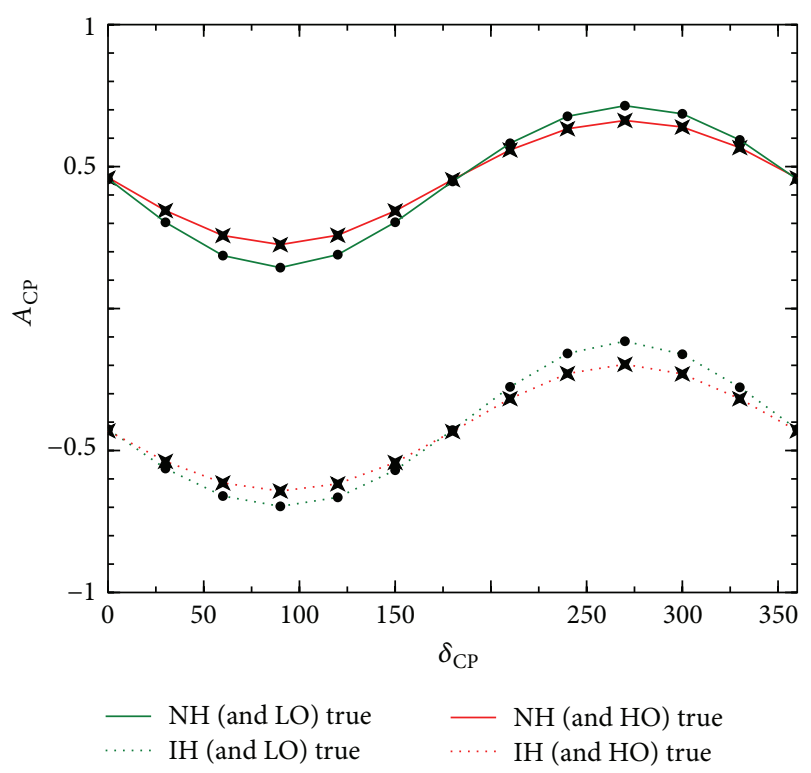

FIGURE 1: CP asymmetry versus $\delta_{\mathrm{CP}}$ at DUNE/LBNE, for both the hierarchies. Red and green solid (dotted) lines are for $\mathrm{NH}(\mathrm{IH})$ with types of curve to distinguish $\mathrm{HO}$ and $\mathrm{LO}$ as the true octant, respectively.

within the $\mathrm{SO}(10)$ model, by using two distinct forms for the lepton CP asymmetry, can be used to break the entanglement. Finally in Section 5, we present our conclusions.

\section{CPV Phase and Octant of $\theta_{23}$}

As discussed above, from Figure 3 of [8], we find that by combining with ND and reactor experiments, CPV sensitivity of LBNE improves more for LO (lower octant) than HO (higher octant), for any assumed true hierarchy. In Figure 1 we plot CP asymmetry,

$$
A_{\mathrm{CP}}=\frac{P\left(\nu_{\mu} \rightarrow v_{e}\right)-P\left(\overline{\nu_{\mu}} \rightarrow \overline{v_{e}}\right)}{P\left(v_{\mu} \rightarrow v_{e}\right)+P\left(\overline{v_{\mu}} \rightarrow \overline{v_{e}}\right)}
$$

as a function of leptonic $\mathrm{CPV}$ phase $\delta_{\mathrm{CP}}$, for $0 \leq \delta_{\mathrm{CP}} \leq$ $2 \pi$. CP asymmetry also depends on the mass hierarchy. For $\mathrm{NH}, \mathrm{CP}$ asymmetry is more in LO than in HO. For $\mathrm{IH}$, $\mathrm{CP}$ asymmetry is more in LO than in HO. In this work we have used above information to calculate dependence of leptogenesis on octant of $\theta_{23}$ and quadrant of CPV phase. From Figure 1 we see that

$$
A_{\mathrm{CP}}(\mathrm{LO})>A_{\mathrm{CP}}(\mathrm{HO}) \text {. }
$$

For a given true hierarchy, there are eight degenerate solutions

$$
\begin{gathered}
\left.\delta_{\mathrm{CP}}(\text { first quadrant })-\theta_{23} \text { (lower octant }\right) \\
\left.\delta_{\mathrm{CP}}(\text { second quadrant })-\theta_{23} \text { (lower octant }\right)
\end{gathered}
$$

$\delta_{\mathrm{CP}}$ (third quadrant) $-\theta_{23}$ (lower octant)

$\delta_{\mathrm{CP}}$ (fourth quadrant) $-\theta_{23}$ (lower octant)

$\delta_{\mathrm{CP}}$ (first quadrant) $-\theta_{23}$ (higher octant)

$\delta_{\mathrm{CP}}\left(\right.$ second quadrant) $-\theta_{23}$ (higher octant)

$\delta_{\mathrm{CP}}$ (third quadrant) $-\theta_{23}$ (higher octant)

$\delta_{\mathrm{CP}}$ (fourth quadrant) $\theta_{23}$ (higher octant).

This eight-fold degeneracy can be viewed as

$$
\text { Quadrant of CPV phase-Octant of } \theta_{23}
$$

entanglement. Out of these eight degenerate solutions, only one should be true solution. To pinpoint one true solution, this entanglement has to be broken. We have shown [8] that sensitivity to discovery potential of CPV at LBNEs in LO is improved more, if data from near detector of LBNEs or from reactor experiments is added to data from FD of LBNEs as shown in Figure 3 of [8]. Therefore 8-fold degeneracy of 2 gets reduced to 4 -fold degeneracy, with our proposal [8]. Hence, following this 4-fold degeneracy still remains to be resolved:

$$
\begin{gathered}
\delta_{\mathrm{CP}}(\text { first quadrant })-\theta_{23}(\mathrm{LO}) \\
\delta_{\mathrm{CP}}(\text { second quadrant })-\theta_{23}(\mathrm{LO}) \\
\delta_{\mathrm{CP}}(\text { third quadrant })-\theta_{23}(\mathrm{LO}) \\
\delta_{\mathrm{CP}}(\text { fourth quadrant })-\theta_{23}(\mathrm{LO}) .
\end{gathered}
$$

The possibility of $\theta_{23}>45^{\circ}$, that is, $\mathrm{HO}$ of $\theta_{23}$, is also considered in this work. In this context the degeneracy is

$$
\begin{gathered}
\delta_{\mathrm{CP}}(\text { first quadrant })-\theta_{23}(\mathrm{HO}) \\
\delta_{\mathrm{CP}}(\text { second quadrant })-\theta_{23}(\mathrm{HO}) \\
\delta_{\mathrm{CP}} \text { (third quadrant) }-\theta_{23}(\mathrm{HO}) \\
\delta_{\mathrm{CP}}(\text { fourth quadrant })-\theta_{23}(\mathrm{HO}) .
\end{gathered}
$$

In this work, we propose that leptogenesis can be used to break above-mentioned 4-fold degeneracy of (5) and (6). It is known that observed baryon asymmetry of the universe (BAU) can be explained via leptogenesis [46-50]. In leptogenesis, the lepton-antilepton asymmetry can be explained, if there are complex Yukawa couplings or complex fermion mass matrices. This in turn arises due to complex leptonic $\mathrm{CPV}$ phases, $\delta_{\mathrm{CP}}$, in fermion mass matrices. If all the other parameters except leptonic $\delta_{\mathrm{CP}}$ phase in the formula for lepton-antilepton asymmetry are fixed, for example, then observed value of BAU from experimental observation can be used to constrain quadrant of $\delta_{\mathrm{CP}}$, and hence 4-fold entanglement of (5) and (6) can be broken. An experimental signature of CP violation associated with the Dirac phase $\delta_{\mathrm{CP}}$, in PMNS matrix [51] can in principle be obtained, by searching for $\mathrm{CP}$ asymmetry in $v$ flavor oscillation. To elucidate this proposal, we consider nonsupersymmetric $\mathrm{SO}(10)$ models, in which 


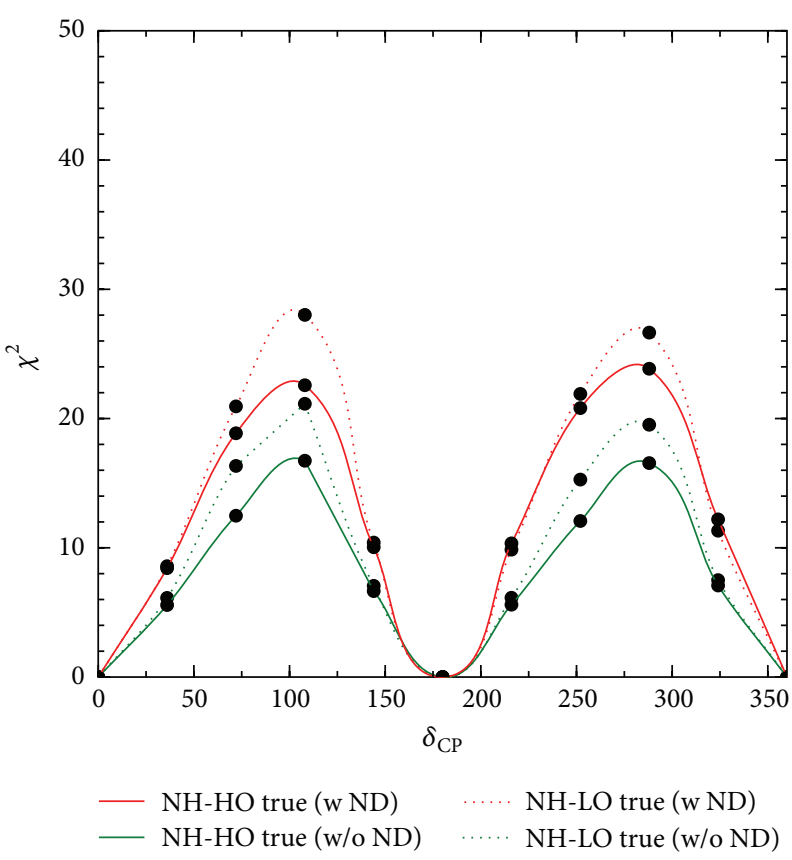

(a)

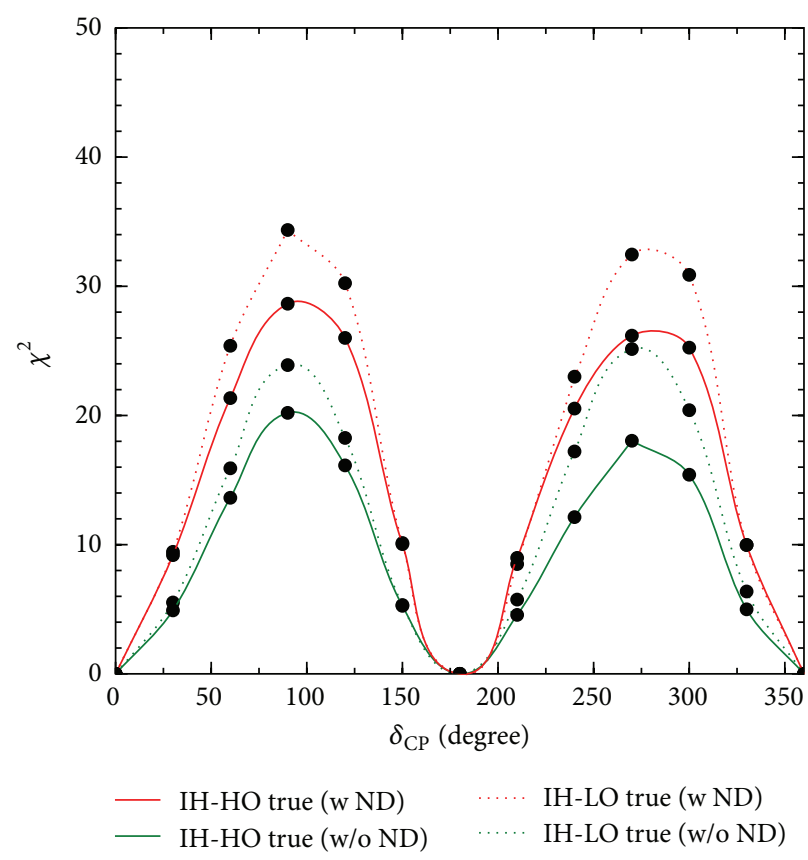

(b)

FIGURE 2: In (a) and (b) $\delta_{\mathrm{CP}}$ versus $\chi^{2}$ sensitivity corresponding to CP discovery potential at LBNEs, for both the hierarchies and octant shown.

BAU arises due to leptogenesis, and this lepton-antilepton asymmetry [52] is generated by the out of equilibrium decay of the right handed, heavy Majorana neutrinos, which form an integral part of seesaw mechanism for neutrino masses and mixing. We consider type I seesaw mechanism, just for simplicity.

\section{Leptogenesis and Baryogenesis Using Type I Seesaw in SO(10) Models}

In grand unified theories like $\mathrm{SO}(10)$, one right-handed heavy Majorana neutrino per generation is added to standard model and they couple with left-handed $v$ via Dirac mass matrix $m_{D}$. When the neutrino mass matrix is diagonalised, we get two eigenvalues, light neutrino $\sim m_{D}^{2} / M_{R}$ and a heavy neutrino state $\sim M_{R}$. This is called type I seesaw mechanism (it may be noted that type I seesaw may be obtained in other theories). Here, decay of the lightest of the three heavy $\mathrm{RH}$ Majorana neutrinos, $M_{1}$, that is $M_{3}, M_{2} \gg M_{1}$, will contribute to $l-\bar{l}$ asymmetry [53-58] (for leptogenesis), that is, $\epsilon_{l}^{\mathrm{CP}}$. It may be noted that some results on leptogenesis in the context of $\mathrm{SO}(10)$ models have been discussed earlier in [59-65]. In the basis where $\mathrm{RH} v$ mass matrix is diagonal, the type I contribution to $\epsilon_{l}^{\mathrm{CP}}$ is given by decay of $M_{1}$

$$
\epsilon_{l}^{\mathrm{CP}}=\frac{\Gamma\left(M_{1} \rightarrow l H\right)-\Gamma\left(M_{1} \rightarrow \bar{l} \bar{H}\right)}{\Gamma\left(M_{1} \rightarrow l H\right)+\Gamma\left(M_{1} \rightarrow \bar{l} \bar{H}\right)},
$$

where $\Gamma\left(M_{1} \rightarrow l H\right)$ means decay rate of heavy Majorana $\mathrm{RH} v$ of mass $M_{1}$ to a lepton and Higgs. We assume a normal mass hierarchy for heavy Majorana neutrinos. In this scenario the lightest of heavy Majorana neutrinos is in thermal equilibrium while the heavier neutrinos, $M_{2}$ and $M_{3}$, decay. Any asymmetry produced by the out of equilibrium decay of $M_{2}$ and $M_{3}$ will be washed away by the lepton number violating interactions mediated by $M_{1}$. Therefore, the final lepton-antilepton asymmetry is given only by the CPviolating decay of $M_{1}$ to standard model leptons $(l)$ and Higgs $(H)$. This contribution is [66]

$$
\epsilon_{l}=-\frac{3 M_{1}}{8 \pi} \frac{\operatorname{Im}\left[\Delta m_{\odot}^{2} R_{12}^{2}+\Delta m_{A}^{2} R_{13}^{2}\right]}{v^{2} \sum\left|R_{1 j}\right|^{2} m_{j}},
$$

where $v$ is the vev of the SM Higgs doublet that breaks the SM gauge group to $U(1)_{e m}$. $R$ is a complex orthogonal matrix with the property that $R R^{T}=1 . R$ can be parameterized as [67]

$$
R=D_{\sqrt{M^{-1}}} Y_{\nu} U D_{\sqrt{K^{-1}}}
$$

where $Y_{v}$ is the matrix of neutrino Yukawa couplings. $v$ is the vacuum expectation value of $v$ Dirac masses. In the flavour basis, where the charged lepton Yukawa matrix, $Y_{e}$, and gauge interactions are flavour-diagonal, $D_{K}=U^{T} K U$, where $K=Y_{v}^{T} M_{R}^{-1} Y_{v}$. $U$ is the PMNS matrix and $M_{R}$ is the $\mathrm{RH}$ neutrino Majorana scale. On the basis of right-handed neutrinos, $D_{M}=\operatorname{Diag}\left(M_{1}, M_{2}, M_{3}\right)$, where $M_{3}, M_{2} \gg M_{1}$. Equation (8) relates the lepton asymmetry to both the solar $\left(\Delta m_{21}^{2}\right)$ and atmospheric $\left(\Delta m_{A}^{2}\right)$ mass squared differences. Thus the magnitude of the matter-antimatter asymmetry can be predicted in terms of low energy oscillation parameters, 


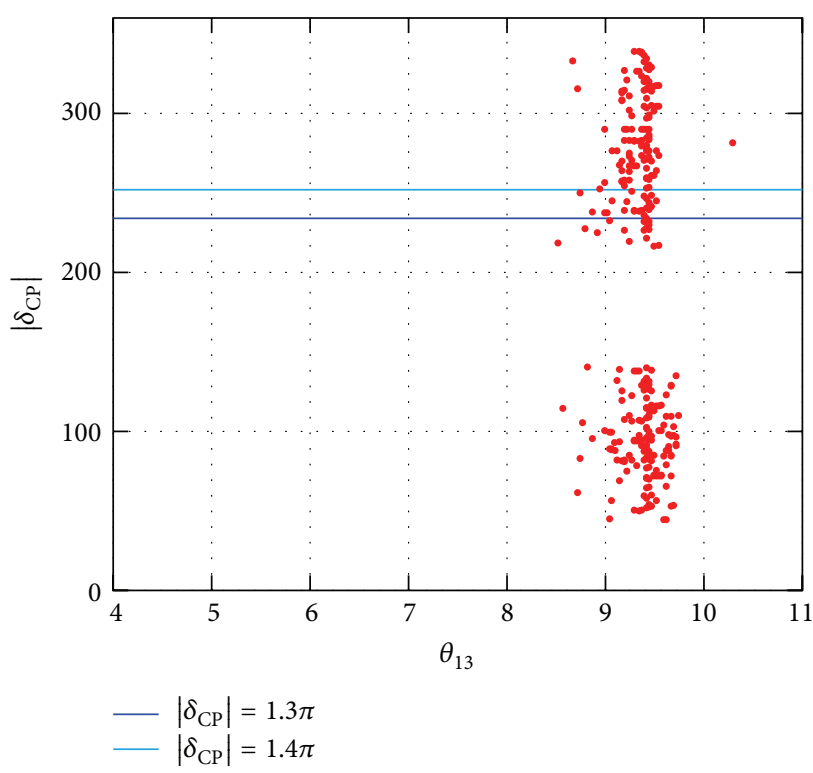

(a)

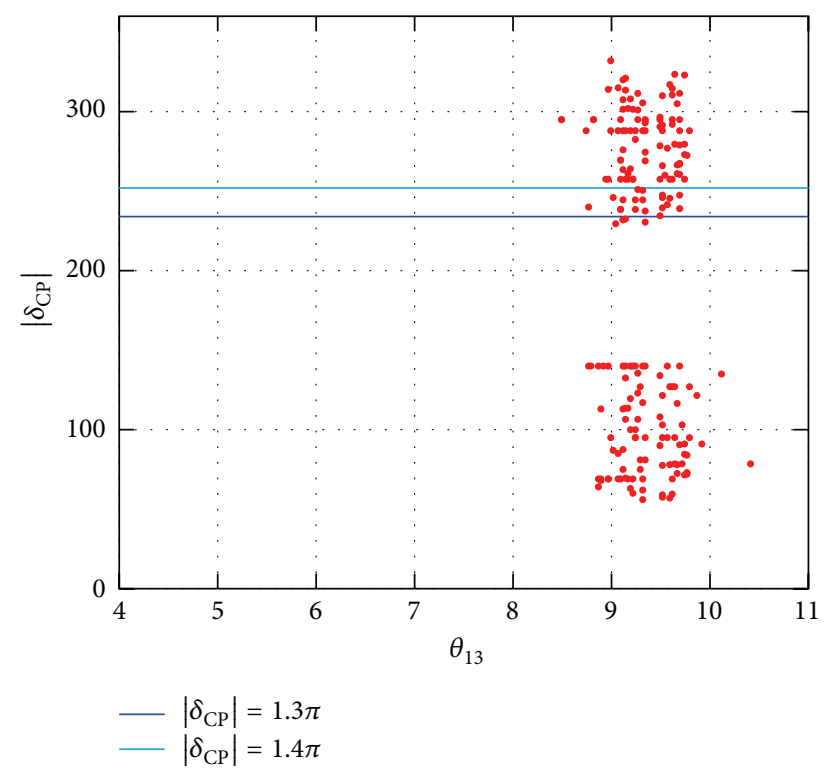

(b)

FIGURE 3: Allowed region constrained by the present BAU bounds, $5.7 \times 10^{-10}<\eta_{B}<6.7 \times 10^{-10}$ for $\delta_{\mathrm{CP}}, \theta_{13}$ for the case when $R$ matrix consists of both $V_{\mathrm{CKM}}$ and $U_{\mathrm{PMNS}}$. The regions are obtained by varying $\delta_{\mathrm{CP}}$ range at $\geq 2 \sigma$ over the corresponding $\chi^{2}$ minima distribution from Figure 2 and $\theta_{13}$ with its experimental values varied within $3 \sigma$. In Figure (a) (Figure (b)) we show the plot for the IH and LO case (IH and $\mathrm{HO}$ case). The blue (cyan) horizontal line represents $\delta_{\mathrm{CP}}=1.3 \pi(1.4 \pi)$ around which the best fit values of CPV phase $\delta_{\mathrm{CP}}$ are assumed to lie.

$\Delta m_{21}^{2}, \Delta m_{A}^{2}$, and a CPV phase. In SO(10) models, the righthanded neutrino $M_{R}$ is generated from the Yukawa coupling of right-handed neutrinos with the Higgs field that breaks the unification or intermediate symmetry down to the standard model [68]. When such a Higgs field takes a VEV, the righthanded neutrinos get a Majorana mass. This happens because lepton number is broken at that scale. It has been discussed in [65] that in the supersymmetric case the mass scale of the right-handed neutrino is similar to the unification scale, $M_{R} \sim M_{U} \sim 10^{16} \mathrm{GeV}$, while in the nonsupersymmetric case the scale of $M_{R}$ is about the intermediate scale, $M_{R} \sim$ $M_{I} \sim 10^{11} \mathrm{GeV}[69,70], M_{I}$ being the scale of the quarklepton symmetry [71].

As has been discussed in [66], the lepton-antilepton asymmetry gets connected to both the solar and the atmospheric mass difference square. Following [72], in this work we choose a basis where the complex orthogonal matrix $R$ takes the form, $R=V_{\mathrm{CKM}} \times U_{\mathrm{PMNS}}$, where $V_{\mathrm{CKM}}$ is the CKM matrix containing the quark mixing angles, and $U_{\text {PMNS }}$ is the PMNS matrix containing neutrino mixing parameters. In equations (9) and (15) of [72], if $V$ is taken as quark mixing matrix (where, in $\mathrm{SO}(10)$ theories, as quarks and leptons appear in same representation, neutrino mixing matrix can be taken to be same as quark mixing matrix at high scales) and $U$ is taken as PMNS matrix at low energies, then we get the relation $R=V_{\mathrm{CKM}} \times U_{\text {PMNs }}$. This assumption can also be justified, as it is well known that quark mixing CKM phase alone is not sufficient to explain the BAU, and leptonic $\mathrm{CPV}$ phase is needed to generate the observed BAU. Here, the matrix $R$ is orthogonal since $R^{\dagger} R=U^{\dagger} V^{\dagger} V U=1$ (because $U^{\dagger} U=V^{\dagger} V=1$ ). Thus both the quark sector (quark mixing angles, phase) and the neutrino sector ( $\nu$ mixing angles and the leptonic CPV phase) appear in the expression for $R$ and in that case $R$ can be parameterized as

$$
\begin{aligned}
& \operatorname{Im} R_{13}^{2} \\
&=-\mathrm{s}\left(2 \delta_{q}\right) \mathrm{c}^{2} 23_{l} \mathrm{c}^{2} 13_{l} \mathrm{~s}^{2} 13_{q} \\
& \quad-2 \mathrm{~s}\left(\delta_{q}\right) \mathrm{c} 13_{q} \mathrm{c} 23_{l} \mathrm{c}^{2} 13_{l} \mathrm{~s} 12_{q} \mathrm{~s} 13_{q} \mathrm{~s} 23_{l} \\
& \quad+2 \mathrm{~s}\left(-\delta_{l}-\delta_{q}\right) \mathrm{c} 12_{q} \mathrm{c} 13_{q} \mathrm{c} 23_{l} \mathrm{c} 13_{l} \mathrm{~s} 13_{\mathrm{q}} \mathrm{s} 13_{l} \\
&-2 \mathrm{~s}\left(\delta_{l}\right) \mathrm{c} 12_{q} \mathrm{c}^{2} 13_{q} \mathrm{c} 13_{l} \mathrm{~s} 12_{q} \mathrm{~s} 23_{l} \mathrm{~s} 13_{l} \\
&-\mathrm{s}\left(2 \delta_{l}\right) \mathrm{c}^{2} 12_{q} \mathrm{c}^{2} 13_{q} \mathrm{~s}^{2} 13_{l} \\
&-2 \mathrm{~s}\left(\delta_{l}\right) \mathrm{c}^{2} 12_{q} \mathrm{c}^{2} 13_{q} \mathrm{~s}^{2} 13_{l} \\
& \operatorname{Im} R_{12}^{2} \\
&= 2 \mathrm{~s}\left(\delta_{q}\right) \mathrm{c} 13_{q} \mathrm{c}^{2} 12_{l} \mathrm{c} 23_{l} \mathrm{~s} 12_{q} \mathrm{~s} 13_{q} \mathrm{~s} 23_{l} \\
&+2 \mathrm{~s}\left(\delta_{q}\right) \mathrm{c} 12_{q} \mathrm{c} 13_{q} \mathrm{c} 12_{l} \mathrm{c} 13_{l} \mathrm{~s} 13_{q} \mathrm{~s} 12_{q} \mathrm{~s} 23_{l} \\
&-\mathrm{s}\left(2 \delta_{q}\right) \mathrm{c}^{2} 12_{l} \mathrm{~s}^{2} 13_{q} \mathrm{~s}^{2} 23_{l} \\
&-2 \mathrm{~s}\left(\delta_{l}-\delta_{q}\right) \mathrm{c} 13_{q} \mathrm{c} 12_{l} \mathrm{c}^{2} 23_{l} \mathrm{~s} 12_{q} \mathrm{~s} 13_{q} \mathrm{~s} 12_{l} \mathrm{~s} 13_{l} \\
&-2 \mathrm{~s}\left(\delta_{l}-\delta_{q}\right) \mathrm{c} 12_{q} \mathrm{c} 13_{q} \mathrm{c} 23_{l} \mathrm{c} 13_{l} \mathrm{~s} 13_{q} \mathrm{~s}^{2} 12_{l} \mathrm{~s} 23_{l} \mathrm{~s}^{2} 12_{q} \mathrm{~s} 12_{l} \mathrm{~s} 23_{l} \mathrm{~s} 13_{l} \\
& \\
& \\
& \\
&
\end{aligned}
$$




$$
\begin{aligned}
& +2 \mathrm{~s}\left(\delta_{l}-2 \delta_{q}\right) \mathrm{c} 12_{l} \mathrm{c} 23_{l} \mathrm{~s}^{2} 13_{q} \mathrm{~s} 12_{l} \mathrm{~s} 23_{l} \mathrm{~s} 13_{l} \\
& -2 \mathrm{~s}\left(\delta_{l}\right) \mathrm{c} 12_{q} \mathrm{c}^{2} 13_{q} \mathrm{c} 13_{l} \mathrm{~s} 12_{q} \mathrm{~s}^{2} 12^{l} \mathrm{~s} 23_{l} \mathrm{~s} 13_{l} \\
& +2 \mathrm{~s}\left(\delta_{l}-\delta_{q}\right) \mathrm{c} 13_{q} \mathrm{c} 12_{l} \mathrm{~s} 12_{q} \mathrm{~s} 12_{q} \mathrm{~s} 12_{l} \mathrm{~s}^{2} 23_{l} \mathrm{~s} 13_{l} \\
& +2 s\left(2 \delta_{l}-2 \delta_{q}\right) \mathrm{c}^{2} 23_{l} \mathrm{~s}^{2} 13_{q} \mathrm{~s}^{2} 12_{l} \mathrm{~s}^{2} 13_{l} \\
& +2 \mathrm{~s}\left(2 \delta_{l}-\delta_{q}\right) \mathrm{c} 13_{q} \mathrm{c} 23_{l} \mathrm{~s} 12_{q} \mathrm{~s} 13_{q} \mathrm{~s}^{2} 12_{l} \mathrm{~s} 23_{l} \mathrm{~s}^{2} 13_{l} \\
& +\mathrm{s}\left(2 \delta_{l}\right) \mathrm{c}^{2} 13_{q} \mathrm{~s}^{2} 12_{q} \mathrm{~s}^{2} 12_{l} \mathrm{~s}^{2} 23_{l} \mathrm{~s}^{2} 13_{l} \\
& R_{11} \\
& \begin{aligned}
= & \mathrm{c} 12_{q} \mathrm{c} 13_{q} \mathrm{c} 12_{l} \mathrm{c} 13_{l}+e^{-i \delta_{q}} \mathrm{~s} 13_{q} \mathrm{~s} 12_{l} \mathrm{~s} 23_{l} \\
& -e^{-i \delta_{l}} e^{-i \delta_{q}} \mathrm{~s} 13^{q} \mathrm{c} 12_{l} \mathrm{c} 23_{l} \mathrm{~s} 13_{l}-\mathrm{c} 13_{q} \mathrm{~s} 12_{q} \mathrm{c} 23_{l} \mathrm{~s} 12_{l} \\
& -e^{-i \delta_{l}} \mathrm{c} 13_{q} \mathrm{~s} 12_{q} \mathrm{c} 12_{l} \mathrm{~s} 23_{l} \mathrm{~s} 13_{l}
\end{aligned} \\
& R_{12} \\
& \begin{aligned}
= & \mathrm{c} 12_{q} \mathrm{c} 13_{q} \mathrm{c} 13_{l} \mathrm{~s} 12_{l}-e^{-i \delta_{q}} \mathrm{~s} 13_{q} \mathrm{c} 12_{l} \mathrm{~s} 23_{l} \\
& -e^{-i \delta_{l}} e^{-i \delta_{q}} \mathrm{c} 23_{l} \mathrm{~s} 12_{l} \mathrm{~s} 13_{l} \mathrm{~s} 13_{q}-\mathrm{c} 13_{q} \mathrm{~s} 12_{q} \mathrm{c} 12_{l} \mathrm{c} 23_{l} \\
& -e^{-i \delta_{l}} \mathrm{c} 13_{q} \mathrm{~s} 12_{q} \mathrm{~s} 12_{l} \mathrm{~s} 23_{l} \mathrm{~s} 13_{l}
\end{aligned} \\
& R_{13} \\
& \begin{aligned}
= & e^{-i \delta_{q}} \mathrm{C} 23_{l} \mathrm{c} 13_{l} \mathrm{~s} 13_{q}-\mathrm{c} 13_{q} \mathrm{c} 13_{l} \mathrm{~s} 12_{q} \mathrm{~s} 23_{l} \\
& -e^{-i \delta_{l}} \mathrm{c} 12_{q} \mathrm{c} 13_{q} \mathrm{~s} 13_{l} .
\end{aligned}
\end{aligned}
$$

Here, $\mathrm{c} 23_{l}, \mathrm{~s} 12_{l}, \mathrm{c} 13_{l}$, and so forth represent the cosine of atmospheric mixing angle, sine of solar mixing angle, and cosine of reactor mixing angle, respectively. Similarly $23_{q}$, $12_{q}$, and $13_{q}$ are the quark mixing angles. $\delta_{l}$ and $\delta_{q}$ are the leptonic CPV phase and quark CPV phase, respectively. Here the $R$ equation presumably holds at the GUT scale but weak scale values can be used in the calculations since it is well known that quark mixing parameters do not change much under renormalization group (RG) evolution in hierarchical mass spectrum of $\mathrm{SO}(10)$ theories. Hence CKM matrix at high scales can be used at low energies also and $U$ is taken as PMNS matrix at low energies. We also consider $R=U_{\text {PMNS }}$, where $R$ matrix consists of $v$ mixing angles and the leptonic $\mathrm{CPV}$ phase. Thus when left-right symmetry is broken at high intermediate mass scale $M_{R}$ in SO(10) theory, CP asymmetry in this case is given by

$$
\epsilon_{l}=-\frac{3 M_{1}}{8 \pi} \frac{\operatorname{Im}\left[\Delta m_{A}^{2} R_{13}^{2}\right]}{v^{2} \sum\left|R_{i j}\right|^{2} m_{j}},
$$

where

$$
\begin{aligned}
& \left|R_{11}\right|^{2}=\cos ^{2}\left(\theta_{12}^{l}\right) \cos ^{2}\left(\theta_{13}^{l}\right), \\
& \left|R_{12}\right|^{2}=\sin ^{2}\left(\theta_{12}^{l}\right) \cos ^{2}\left(\theta_{13}^{l}\right),
\end{aligned}
$$

$$
\begin{aligned}
& \left|R_{13}\right|^{2}=\cos ^{2}\left(\delta_{l}\right) \sin ^{2}\left(\theta_{13}^{l}\right)+\sin ^{2}\left(\delta_{l}\right) \sin ^{2}\left(\theta_{13}^{l}\right), \\
& \operatorname{Im} R_{13}^{2}=-\sin ^{2}\left(2 \delta_{l}\right) \sin ^{2}\left(\theta_{13}^{l}\right) .
\end{aligned}
$$

The neutrino oscillation data used in our numerical calculations are summarised as follows [31,38-42]:

$$
\begin{aligned}
\Delta m_{21}^{2}\left[10^{-5} \mathrm{eV}^{2}\right] & =7.60_{-0.18}^{+0.19} \\
\left|\Delta m_{31}^{2}\right|\left[10^{-3} \mathrm{eV}^{2}\right] & =2.48_{-0.07}^{+0.05}\left(2.38_{-0.06}^{+0.05}\right) \\
\sin ^{2} \theta_{12} & =0.323 \pm 0.016 \\
\sin ^{2} \theta_{23} & =0.567_{-0.124}^{+0.032}\left(0.573_{-0.039}^{+0.025}\right) \\
\sin ^{2} \theta_{13} & =0.0226 \\
& \pm 0.0012(0.0229 \pm 0.0012) .
\end{aligned}
$$

For $\Delta m_{31}^{2}, \sin ^{2} \theta_{23}$, and $\sin ^{2} \theta_{13}$, the quantities inside the bracket correspond to inverted neutrino mass hierarchy and those outside the bracket correspond to normal mass hierarchy. The errors are within the $1 \sigma$ range of the $v$ oscillation parameters. It may be noted that some results on neutrino masses and mixing using updated values of running quark and lepton masses in SUSY SO(10) have also been presented in [73]. Though we consider 3-flavour neutrino scenario, 4flavour neutrinos with sterile neutrinos as fourth flavour are also possible [74]. It is worth mentioning that $\nu$ masses and mixing can lead to charged lepton flavor violation in grand unified theories like $\mathrm{SO}(10)$ [75].

The origin of the baryon asymmetry in the universe (baryogenesis) is a very interesting topic of current research. A well known mechanism is the baryogenesis via leptogenesis, where the out of equilibrium decays of heavy righthanded Majorana neutrinos produce a lepton asymmetry which is transformed into a baryon asymmetry [72] by electroweak sphaleron processes [76-79]. Lepton asymmetry is partially converted to baryon asymmetry through $\mathrm{B}+$ $\mathrm{L}$ violating sphaleron interactions [80]. As proposed in [65], a baryon asymmetry can be generated from a lepton asymmetry. The baryon asymmetry is defined as

$$
Y_{B}=\frac{n_{B}-n_{\bar{B}}}{s}=\frac{n_{B}-n_{\bar{B}}}{7 n_{\gamma}}=\frac{\eta_{B}}{7}
$$

where $n_{B}, n_{\bar{B}}$, and $n_{\gamma}$ are number densities of baryons, antibaryons, and photons, respectively, $s$ is the entropy density, $\eta$ is the baryon to photon ratio, and $5.7 \times 10^{-10} \leq \eta_{B} \leq$ $6.7 \times 10^{-10}(95 \% \mathrm{CL})$ [81]. The lepton number is converted into the baryon number through electroweak sphaleron process [76-79]:

$$
Y_{B}=\frac{a}{a-1} Y_{L}, \quad a=\frac{8 N_{F}+4 N_{H}}{22 N_{F}+13 N_{H}},
$$


where $N_{F}$ is the number of families and $N_{H}$ is the number of light Higgs doublets. In case of SM, $N_{F}=3$ and $N_{H}=1$. The lepton asymmetry is as follows:

$$
Y_{L}=d \frac{\epsilon_{l}}{g^{*}}
$$

$d$ is a dilution factor and $g^{*}=106.75$ in the standard case [65] is the effective number of light degrees of freedom in the theory. The dilution factor $d$ [65] is $d=0.24 / k(\ln k)^{0.6}$ for $k \geq 10$ and $d=1 / 2 k, d=1$ for $1 \leq k \leq 10$, and $0 \leq k \leq 1$, respectively, where the parameter $k$ [65] is $k=\left(M_{P} / 1.7 v^{2} 32 \pi \sqrt{g^{*}}\right)\left(\left(M_{D}^{\dagger} M_{D}\right)_{11} / M_{1}\right)$; here $M_{P}$ is the Planck mass. We have used the form of Dirac neutrino mass matrix $M_{D}$ from [37].

\section{Calculations, Results, and Discussion}

For the purpose of calculations, we use the current experimental data for three neutrino mixing angles as inputs, which are given at $1 \sigma-3 \sigma$ C.L, as presented in [31, 38-42]. Here, we perform numerical analysis for both the hierarchies and octants. We explore the baryon asymmetry of the universe within nonsupersymmetric $\mathrm{SO}(10)$ models [37] using (7)(16) of the two hierarchies $(\mathrm{NH}$ and $\mathrm{IH})$, two octants $\mathrm{LO}$ and $\mathrm{HO}, \mathrm{w} \mathrm{ND}, \mathrm{w} / \mathrm{o} \mathrm{ND}$ (with and without near detector), and $\delta_{\mathrm{CP}}$ range at $\geq 2 \sigma$ over the corresponding distribution of $\chi^{2}$-minima (for maximum sensitivity from Figures 2(a) and 2(b), for which the CP discovery potential of the DUNE is maximum). For our purpose, we shall carry out general scanning of the parameters: $\delta_{\mathrm{CP}}$ range at $\geq 2 \sigma$ (from Figures 2(a) and 2(b)), $\theta_{13}$ at its $3 \sigma \mathrm{CL}$, and $\Delta m_{31}^{2}$ at its $3 \sigma \mathrm{CL}$ using the data given by the oscillation experiments [1-6, 31, 38-42]. In this calculation, we have chosen $M_{1} \sim 2.154 \times 10^{11} \mathrm{GeV}$. Also we note that, for values of $M_{1}<2.154 \times 10^{11} \mathrm{GeV}$, the baryon asymmetry becomes lower than the observed value. We scan the parameter space for $\mathrm{IH}$ and $\mathrm{HO} / \mathrm{LO}$ in the light of recent ratio of the baryon to photon density bounds, $5.7 \times 10^{-10} \leq$ $\eta_{B} \leq 6.7 \times 10^{-10}(\mathrm{CMB})[81]$ in the following ranges:

$$
\begin{aligned}
\delta_{\mathrm{CP}} & \in[0,2 \pi] \\
\theta_{13} & \in\left[7.8^{\circ}, 9.9^{\circ}\right] \\
\Delta m_{31}^{2} & \in\left[-2.54 * 10^{-3},-2.20 * 10^{-3}\right] \mathrm{eV}^{2} .
\end{aligned}
$$

Similarly constrained by the present BAU bounds we perform random scans for the following range of parameters in $\mathrm{NH}$, $\mathrm{HO} / \mathrm{LO}$ case:

$$
\begin{aligned}
\delta_{\mathrm{CP}} & \in[0,2 \pi] \\
\theta_{13} & \in\left[7.7^{\circ}, 9.9^{\circ}\right] \\
\Delta m_{31}^{2} & \in\left[2.30 * 10^{-3}, 2.65 * 10^{-3}\right] \mathrm{eV}^{2} .
\end{aligned}
$$

We find that the updated BAU limit [81] together with a large $\theta_{13}[1-7]$ puts significant constraints on the $\delta_{\mathrm{CP}}-\theta_{13}$ parameter space in the IH and LO case. As can be seen from Figure 3(a), a part of the parameter space survives for $\delta_{\mathrm{CP}} \simeq 1.3-1.4 \pi$ in the IH and LO case as allowed by the current BAU constraint
$5.7 \times 10^{-10} \leq \eta_{B} \leq 6.7 \times 10^{-10}(\mathrm{CMB})$, corresponding to $\theta_{13}$ around $8.9^{\circ}-9.5^{\circ}$. This leads to the conclusion that the parameter space for the best fit values of $\delta_{\mathrm{CP}} \simeq 1.3-1.4 \pi$ is allowed by the present BAU constraint. The allowed regions in Figure 3(a) for the lower quadrant ( 0 to $\pi$ ) require $\delta_{\mathrm{CP}}$ spectra, that is, $\delta_{\mathrm{CP}}$ to be equal to $88^{\circ}$ for $\theta_{13}$ around $9.09^{\circ}$ to $9.2^{\circ}, 9.25^{\circ}, 9.35^{\circ}$ to $9.5^{\circ}$, and $9.6^{\circ}$ to $9.65^{\circ}$. Almost continuous values of $\delta_{\mathrm{CP}}$ ranging from $50^{\circ}$ to $140^{\circ}$ are allowed for $\theta_{13}$, $9.3^{\circ}$ to $9.5^{\circ}$. For $\theta_{13}$ around $9.7^{\circ}$, the values of $\delta_{\mathrm{CP}}$ mostly favoured are $50^{\circ}, 69^{\circ}, 88^{\circ}, 90^{\circ}, 95^{\circ}, 99^{\circ}, 120^{\circ}$, and $130^{\circ}$. The allowed region in the upper quadrant ( $\pi$ to $2 \pi$ ) necessitates $\delta_{\mathrm{CP}}$ to be around $276.5^{\circ}$, for $\theta_{13}$ around $9.06^{\circ}$ to $9.12^{\circ}, 9.3^{\circ}$ to $9.45^{\circ}$, and $9.517^{\circ}$ as allowed by the current BAU bounds. Also $\delta_{\mathrm{CP}}=290^{\circ}$ exists for $\theta_{13}$ around $9^{\circ}, 9.15^{\circ}$ to $9.3^{\circ}$, and $9.35^{\circ}$ to $9.5^{\circ}$. Almost continuous $\delta_{\mathrm{CP}}$ ranging from $230^{\circ}$ to $340^{\circ}$ are allowed for $\theta_{13}$ around $9.4^{\circ}$.

For $\mathrm{IH}$ and $\mathrm{HO}$ case in Figure 3(b), a part of the $\delta_{\mathrm{CP}}-\theta_{13}$ parameter space exists for $\delta_{\mathrm{CP}} \simeq 1.3-1.4 \pi$ constrained by the current BAU limits for $\theta_{13}$ around $9^{\circ}, 9.097^{\circ}, 9.1^{\circ}, 9.2^{\circ}, 9.3^{\circ}$, $9.5^{\circ}, 9.55^{\circ}, 9.6^{\circ}$, and $9.7^{\circ}$. Thus the parameter space for the best fit values of $\delta_{\mathrm{CP}} \simeq 1.3-1.4 \pi$ is allowed by the present $\mathrm{BAU}$ constraint [81]. Figure 3(b) reveals that the allowed regions for the lower quadrant ( 0 to $\pi$ ) require $\delta_{\mathrm{CP}}$ to be around $69^{\circ}$, for $\theta_{13} \sim 8.86^{\circ}-8.896^{\circ}, 9.06^{\circ}-9.22^{\circ}, 9.32^{\circ}$, and $9.61^{\circ}$. There also exists $\delta_{\mathrm{CP}}=88^{\circ}$ which constrains $\theta_{13}$ to be around $9.0^{\circ}$ to $9.1^{\circ}$. Also $\delta_{\mathrm{CP}}=95^{\circ}$ survives for $\theta_{13}$ around $8.99^{\circ}$, $9.24^{\circ}, 9.352^{\circ}, 9.5^{\circ}-9.64^{\circ}$, and $9.79^{\circ}$. Almost continuous values of $\delta_{\mathrm{CP}}$ ranging from $50^{\circ}$ to $140^{\circ}$ are allowed for $\theta_{13}$ varying from $8.75^{\circ}$ to $9.75^{\circ}$. In the upper quadrant ( $\pi$ to $2 \pi$ ) for the present updated BAU constraint, the allowed region of $\delta_{\mathrm{CP}}$ parameter space becomes constrained with $\delta_{\mathrm{CP}}=257.5^{\circ}$, for $\theta_{13}$ around $8.96^{\circ}, 9.0974^{\circ}$ to $9.22^{\circ}, 9.5^{\circ}, 9.6^{\circ}$, and $9.74^{\circ}$. Also the BAU constraints necessitate $\delta_{\mathrm{CP}}$ to be around $288^{\circ}$, for $\theta_{13}$ around $9^{\circ}, 9.06^{\circ}$ to $9.35^{\circ}, 9.5^{\circ}, 9.69^{\circ}$, and $9.8^{\circ}$ as allowed by the current BAU bounds. Also $\delta_{\mathrm{CP}}=295^{\circ}$ survives for $\theta_{13}$ around $9.09^{\circ}, 9.26^{\circ}, 9.34^{\circ}, 9.5^{\circ}, 9.6^{\circ}$, and $9.69^{\circ}$. Almost continuous $\delta_{\mathrm{CP}}$ ranging from $232^{\circ}$ to $340^{\circ}$ are allowed for $\theta_{13}$ around $8.9^{\circ}$ and $9.65^{\circ}$.

The constraints imposed on the $\delta_{\mathrm{CP}}$ and $\theta_{13}$ parameter in $\mathrm{NH}, \mathrm{HO} / \mathrm{LO}$ space are found to be more severe as compared to $\mathrm{IH}$ and $\mathrm{HO} / \mathrm{LO}$ space. For, $\mathrm{NH}$ and $\mathrm{LO}$ only a particular value of $\mathrm{CP}$ violating phase, $\delta_{\mathrm{CP}}=258.5^{\circ}$ corresponding to $\theta_{13}=9.02375^{\circ}$, is consistent with the BAU constraint. From our analysis we find that for $\mathrm{NH}$ and $\mathrm{HO}$ case we are unable to resolve the entanglement of the quadrant of $\delta_{\mathrm{CP}}$ and octant of $\theta_{23}$ since no point in the parameter space $\left(\delta_{\mathrm{CP}}, \theta_{13}\right)$ is consistent with the recent ratio of baryon to photon density bounds, $5.7 \times 10^{-10}<\eta_{B}<6.7 \times 10^{-10}$. Therefore, this indicates that $\mathrm{IH}$ is the most favoured hierarchy for breaking the 4-fold degeneracy of (5) and (6). All of the analysis presented above is for the case when $R$ matrix consists of both $V_{\mathrm{CKM}}$ and $U_{\mathrm{PMNS}}$.

No points in the $\delta_{\mathrm{CP}}-\theta_{13}$ parameter space, consistent with the BAU constraint, are able to break the entanglement of the quadrant of $\delta_{\mathrm{CP}}$ and octant of $\theta_{23}$, when $R$ matrix consists of $U_{\text {PMNS }}$ only.

In Figures 4 and 5 we display the allowed $3 \mathrm{D}$ space $\left(\delta_{\mathrm{CP}}\right.$, $\theta_{13}$, and $\Delta m_{31}^{2}$ ) for breaking the 4-fold degeneracy of (5) and 
TABLE 1: The summary of our calculated values of $\delta_{\mathrm{CP}}, \theta_{13}$, and $\Delta m_{31}^{2}$ in case of IH and LO for $R_{1 j}$ elements of $R$ matrix comprising $U_{\mathrm{PMNS}}$ and $V_{\mathrm{CKM}}$.

\begin{tabular}{lcccc}
\hline Serial number $\begin{array}{c}\text { Leptonic CPV } \\
\text { phase } \delta_{\mathrm{CP}}\end{array}$ & {$\left[\Delta m_{31}^{2}\right] \mathrm{eV}^{2}$} & $\theta_{13}$ & Quadrant of $\delta_{\mathrm{CP}}$ \\
\hline 1 & $\delta_{\mathrm{CP}}=88^{\circ}$ & {$\left[-2.54 * 10^{-3},-2.21 * 10^{-3}\right]$} & $9.0417^{\circ}, 9.0697^{\circ}, 9.0974^{\circ}, 9.3917^{\circ}, 9.4417^{\circ}, 9.6167^{\circ}, 9.6417^{\circ}$ & $\mathrm{I}$ \\
2 & $\delta_{\mathrm{CP}}=276.5^{\circ}$ & {$\left[-2.45 * 10^{-3},-2.21 * 10^{-3}\right]$} & $9.0667^{\circ}, 9.0974^{\circ}, 9.3167^{\circ}-9.4417^{\circ}, 9.5167^{\circ}$ & $\mathrm{IV}^{\circ}$ \\
3 & $\delta_{\mathrm{CP}}=290^{\circ}$ & {$\left[-2.42 * 10^{-3},-2.54 * 10^{-3}\right]$} & $9^{\circ}, 9.1817^{\circ}, 9.2667^{\circ}, 9.3667^{\circ}-9.4417^{\circ}$ & $\mathrm{IV}$ \\
\hline
\end{tabular}

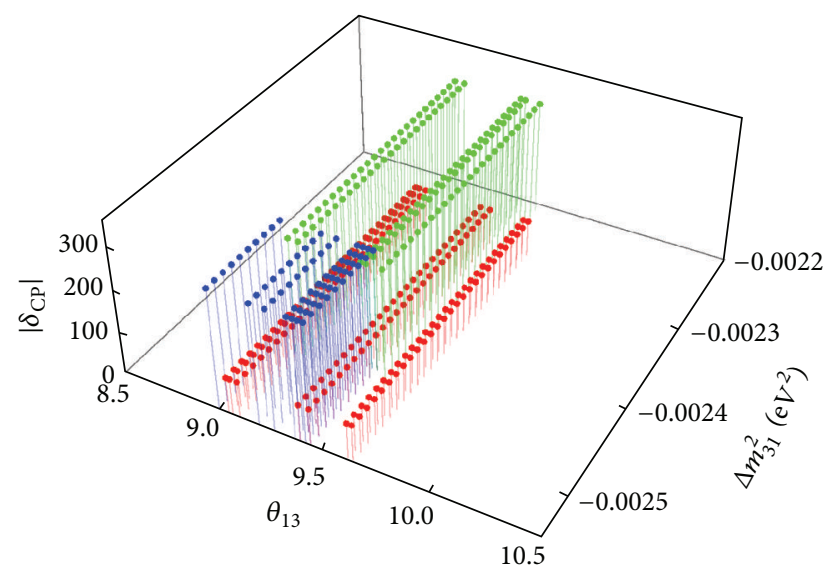

$$
\begin{aligned}
& \delta_{\mathrm{CP}}=276.5^{\circ} \\
& \delta_{\mathrm{CP}}=88^{\circ} \\
& \text { - } \delta_{\mathrm{CP}}=290^{\circ}
\end{aligned}
$$

FIGURE 4: Allowed region constrained by the present BAU bounds, $5.7 \times 10^{-10}<\eta_{B}<6.7 \times 10^{-10}$ for $\delta_{\mathrm{CP}}, \theta_{13}$, and $\Delta m_{31}^{2}$ for the case when $R$ matrix consists of both $V_{\mathrm{CKM}}$ and $U_{\text {PMNS }}$. The regions are obtained by varying $\delta_{\mathrm{CP}}$ range at $\geq 2 \sigma$ over the corresponding $\chi^{2}$ minima distribution from Figure 2 and $\theta_{13}$ with its experimental values varied within $3 \sigma$ and $\Delta m_{31}^{2}$ at its $3 \sigma$ C.L. The results of our calculation are presented for $\mathrm{IH}$ and $\mathrm{LO}$ case.

(6) by varying the leptonic $\delta_{\mathrm{CP}}$ phase in all of its possible range $[0-2 \pi]$ at $\geq 2 \sigma$ (from Figure 2 ), the mixing parameter $\theta_{13}$ within its $3 \sigma$ level, and $\Delta m_{31}^{2}$ at its $3 \sigma$ C.L [6].

From Figure 4 one can easily see the favoured values of $\delta_{\mathrm{CP}}, \theta_{13}$, and $\Delta m_{31}^{2}$ for IH and LO case, allowed by the updated recent ratio of photon density to baryon density bounds, $5.7 \times$ $10^{-10}<\eta_{B}<6.7 \times 10^{-10}$ (shown in Table 1 ).

For $\mathrm{IH}$ and $\mathrm{HO}$ case, the results of our numerical analysis are shown in Figure 5 which shows allowed $\left(\delta_{\mathrm{CP}}, \theta_{13}, \Delta m_{31}^{2}\right)$ space as allowed by the current BAU bounds. The values of $\delta_{\mathrm{CP}}, \theta_{13}$, and $\Delta m_{31}^{2}$ which are favoured simultaneously consistent with $\eta_{B}$ constraints, $5.7 \times 10^{-10}<\eta_{B}<6.7 \times 10^{-10}$ [81], are shown in Table 2 . For $\mathrm{NH}$ case we get only one point as shown in

$\mathrm{NH}, \mathrm{HO}$, III quadrant of Leptonic $\delta_{\mathrm{CP}}$ phase $\delta_{\mathrm{CP}}$ $=258.4^{\circ}$ or $1.436 \pi$.

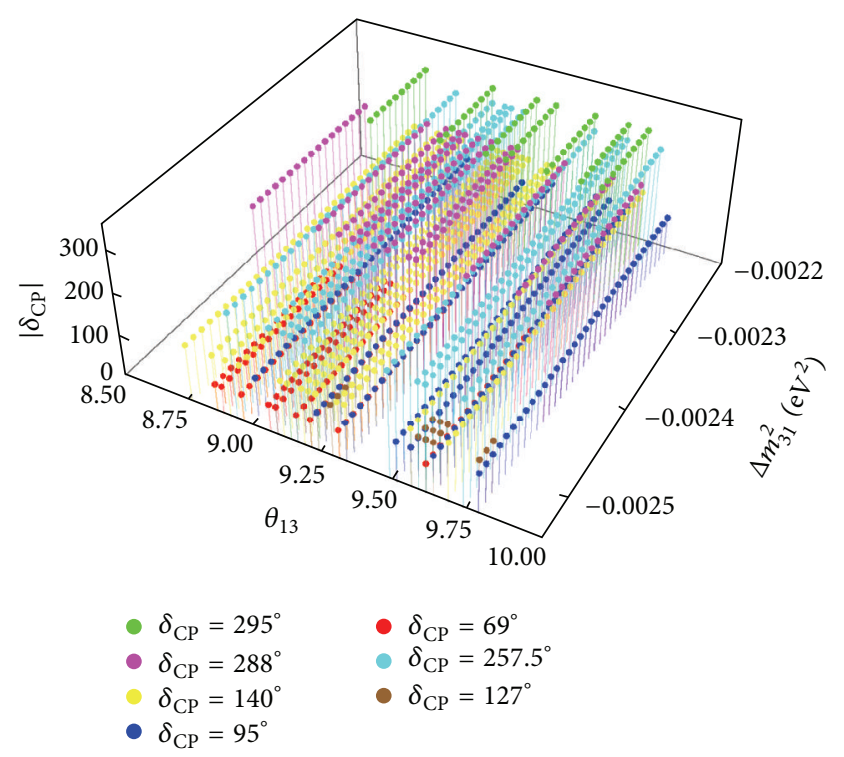

FIGURE 5: Allowed 3D region constrained by the present BAU bounds, $5.7 \times 10^{-10}<\eta_{B}<6.7 \times 10^{-10}$ for $\delta_{\mathrm{CP}}, \theta_{13}$, and $\Delta m_{31}^{2}$ for the case when $R$ matrix consists of both $V_{\mathrm{CKM}}$ and $U_{\mathrm{PMNS}}$. The regions are obtained by varying $\delta_{\mathrm{CP}}$ range at $\geq 2 \sigma$ over the corresponding $\chi^{2}$ minima distribution from Figure $2, \theta_{13}$ with its experimental values varied within $3 \sigma$ from its central values, and $\Delta m_{31}^{2}$ at its $3 \sigma \mathrm{CL}$. The results of our calculation are presented for $\mathrm{IH}$ and $\mathrm{HO}$ case.

\section{Conclusion}

Measuring CP violation in the lepton sector is one of the most challenging tasks today. A systematic study of the CP sensitivity of the current and upcoming LBNE/DUNE is done in our earlier work [8] which may help a precision measurement of leptonic $\delta_{\mathrm{CP}}$ phase. In this work, we studied how the entanglement of the quadrant of leptonic CPV phase and octant of atmospheric mixing angle $\theta_{23}$ at LBNE/DUNE can be broken via leptogenesis and baryogenesis. Here, we have considered the effect of ND only in LBNE, on sensitivity of CPV phase measurement, but similar conclusions would hold for the effect of reactor experiments as well. This study is done for both the octants and hierarchies. We considered two cases of fermion rotation matrix, PMNS only and CKM + PMNS. Following the results of [8], the enhancement of CPV sensitivity with respect to its quadrant is utilized here to calculate the values of lepton-antilepton symmetry. Then, this is used to calculate the value of BAU. This is an era of precision measurements in neutrino physics. We therefore 
TABLE 2: The summary of our calculated values of $\delta_{\mathrm{CP}}, \theta_{13}$, and $\Delta m_{31}^{2}$ in case of IH and HO for $R_{1 j}$ elements of $R$ matrix comprising $U_{\mathrm{PMNS}}$ and $V_{\mathrm{CKM}}$.

\begin{tabular}{|c|c|c|c|c|}
\hline Serial number & $\delta_{\mathrm{CP}}$ & {$\left[\Delta m_{31}^{2}\right] \mathrm{eV}^{2}$} & $\theta_{13}$ & Quadrant of $\delta_{\mathrm{CP}}$ \\
\hline 1 & $95^{\circ}$ & {$\left[-2.54 * 10^{-3},-2.21 * 10^{-3}\right]$} & $8.9917^{\circ}, 9.2417^{\circ}, 9.352^{\circ}, 9.5167^{\circ}, 9.567^{\circ}, 9.6417^{\circ}, 9.7917^{\circ}$ & II \\
\hline 2 & $69^{\circ}$ & {$\left[-2.54 * 10^{-3},-2.38 * 10^{-3}\right]$} & $\begin{array}{c}8.8667^{\circ}, 8.8917^{\circ}, 8.9667^{\circ}, 9.0667^{\circ}, 9.0917^{\circ}, 9.1667^{\circ}, 9.2167^{\circ}, \\
9.3167^{\circ}, 9.6167^{\circ}\end{array}$ & I \\
\hline 3 & $140^{\circ}$ & {$\left[-2.54 * 10^{-3},-2.21 * 10^{-3}\right]$} & $\begin{array}{c}8.7667^{\circ}, 8.8667^{\circ}, 8.9167^{\circ}, 8.9667^{\circ}, 9.1167^{\circ}, 9.1417^{\circ} \\
9.1917^{\circ}-9.2417^{\circ}, 9.3167^{\circ}, 9.3417^{\circ}, 9.5667^{\circ}, 9.6917^{\circ}\end{array}$ & II \\
\hline 4 & $257.5^{\circ}$ & {$\left[-2.54 * 10^{-3},-2.21 * 10^{-3}\right]$} & $\begin{array}{c}8.9667^{\circ}, 9.0974^{\circ}, 9.1417^{\circ}, 9.1667^{\circ}, 9.2167^{\circ}, 9.4917^{\circ}, 9.5917^{\circ}, \\
9.6167^{\circ}, 9.7417^{\circ}\end{array}$ & III \\
\hline 5 & $295^{\circ}$ & {$\left[-2.3 * 10^{-3},-2.21 * 10^{-3}\right]$} & $8.4917^{\circ}, 8.8167^{\circ}, 9.0917^{\circ}, 9.2667^{\circ}, 9.4917^{\circ}, 9.6167^{\circ}$ & IV \\
\hline 6 & $288^{\circ}$ & {$\left[-2.44 * 10^{-3},-2.28 * 10^{-3}\right]$} & $\begin{array}{c}8.7817^{\circ}, 8.9917^{\circ}, 9.0667^{\circ}, 9.1167^{\circ}, 9.1417^{\circ}, 9.1917^{\circ}, 9.2417^{\circ}, \\
9.3167^{\circ}, 9.3417^{\circ}, 9.5167^{\circ}, 9.6917^{\circ}, 9.7917^{\circ}\end{array}$ & IV \\
\hline
\end{tabular}

considered variation of $\Delta m_{31}^{2}$ and $\theta_{13}$ within its $3 \sigma$ range from their central values. We calculated baryon to photon ratio and compared with its experimentally known best fit value.

It has been found [65] that, in SUSY theories, $Y_{B}$ does not lie in the allowed range of the order of $10^{-10}$. Nonsupersymmetric $\mathrm{SO}(10)$ model is favoured for baryogenesis with respect to the supersymmetric model. In the nonsupersymmetric model [37], we find that the baryon asymmetry is around the required order of magnitude $Y_{B} \sim 10^{-10}$. We have made a complete numerical analysis of the 3-dimensional parameters, $\delta_{\mathrm{CP}}, \theta_{13}$, and $\Delta m_{31}^{2}$, that encode the breaking of the entanglement of the quadrant of CPV phase and octant of $\theta_{23}$ in the presence of the latest constraints on $\left|\eta_{B}\right|, 5.7 \times$ $10^{-10}<\eta_{B}<6.7 \times 10^{-10}$, by taking neutrino oscillation mixing and mass scales as indicated by the experiments. By allowing $\delta_{\mathrm{CP}}$ range to vary within $[0-2 \pi]$ interval at $\geq 2 \sigma$ over the $\chi^{2}$-minima distribution from Figure 2 , we have studied the absolute values of both $\theta_{13}$ and $\delta_{\mathrm{CP}}$ parameters in order to break the 4-fold degeneracy of (5) and (6).

The current data shows a preference of $\delta_{\mathrm{CP}}$ towards $1.5 \pi$. From our analysis, one of the leptonic CPV phases determined in $\mathrm{IH}$ and $\mathrm{LO}$ of $\theta_{23}$ case is $\delta_{\mathrm{CP}}=276.5^{\circ}$ or $1.536 \pi$ corresponding to $\theta_{13} \sim 9.0667^{\circ}, 9.0974^{\circ}, 9.3167^{\circ}-9.4417^{\circ}$, $9.5167^{\circ}$, and $\Delta m_{31}^{2} \in\left[-2.45 * 10^{-3},-2.21 * 10^{-3}\right] \mathrm{eV}^{2}$ which is near the preferred data $\delta_{\mathrm{CP}}=(3 / 2) \pi$ reported in [4345]. The current analysis reveals that our calculated value of $\delta_{\mathrm{CP}}=1.43 \pi$ of $\mathrm{IH}$ and $\mathrm{HO}$ of $\theta_{23}$ case is close to the best fit value of $\delta_{\mathrm{CP}}=1.48 \pi$ for inverted ordering from global fit results $[31,38-42]$ and also $\delta_{\mathrm{CP}}=1.436 \pi$ of $\mathrm{NH}$ and $\mathrm{HO}$ of $\theta_{23}$ case is favoured with the recent hint of $\delta_{\mathrm{CP}}=1.41 \pi$ for normal hierarchy [31, 38-42].

The main results of this work are presented in Tables 1 and 2 and (19) which show that leptonic CPV phases in all the four quadrants are allowed which lie within the constraints of present BAU. These values also contain the best fit values of leptonic CPV phase as discussed earlier.

These results could be important, as the quadrant of leptonic CPV phase and octant of atmospheric mixing angle $\theta_{23}$ are yet not fixed experimentally. Also, they are significant in context of precision measurements of neutrino oscillation parameters, specially the leptonic CPV phase, $\Delta m_{31}^{2}$, and the reactor angle $\theta_{13}$.
Future experiments like DUNE/LBNEs and HyperKamiokande [82] looking for the leptonic CPV phase $\delta_{\mathrm{CP}}$ together with an improvement in the precision determination on the mixing angles would certainly provide worthy information to support or rule out the scenario presented in this work for breaking the entanglement of quadrant of CPV phase and octant of $\theta_{23}$.

\section{Competing Interests}

The authors declare that they have no competing interests.

\section{Acknowledgments}

Gayatri Ghosh would like to thank UGC, India, for providing RFSMS Fellowship to her, during which this work was done. Kalpana Bora thanks DST-SERB, Govt. of India, for financial support through a project. Debajyoti Dutta acknowledges the support from DAE Neutrino Project at HRI.

\section{References}

[1] G. Fogli, E. Lisi, A. Marrone, D. Montanino, A. Palazzo, and A. M. Rotunno, "Global analysis of neutrino masses, mixings, and phases: entering the era of leptonic $C P$ violation searches," Physical Review D, vol. 86, Article ID 013012, 2012.

[2] F. P. An, J. Z. Bai, A. B. Balantekin et al., "Observation of electron-antineutrino disappearance at Daya Bay," Physical Review Letters, vol. 108, no. 17, Article ID 171803, 7 pages, 2012.

[3] F. P. An, Q. An, J. Z. Bai et al., "Improved measurement of electron antineutrino disappearance at Daya Bay," Chinese Physics C, vol. 37, no. 1, Article ID 011001, 2013.

[4] J. K. Ahn, S. Chebotaryov, J. H. Choi et al., "Observation of reactor electron antineutrinos disappearance in the RENO experiment," Physical Review Letters, vol. 108, no. 19, Article ID 191802, 2012.

[5] Y. Abe, C. Aberle, T. Akiri et al., "Indication of reactor $\bar{v}_{e}$ disappearance in the double Chooz experiment," Physical Review Letters, vol. 108, no. 13, Article ID 131801, 2012.

[6] D. Forero, M. Tórtola, and J. W. F. Valle, "Global status of neutrino oscillation parameters after Neutrino-2012," Physical Review D, vol. 86, no. 7, Article ID 073012, 8 pages, 2012. 
[7] M. Gonzalez-Garcia, M. Maltoni, J. Salvado, and T. Schwetz, "Global fit to three neutrino mixing: critical look at present precision," Journal of High Energy Physics, vol. 2012, article 123, 2012.

[8] D. Dutta and K. Bora, "Probing CP violation at LBNE with reactor experiments," Modern Physics Letters A, vol. 30, no. 7, Article ID 1550017, 13 pages, 2015.

[9] M. Ghosh, P. Ghoshal, S. Goswami, and S. K. Raut, "Evidence for leptonic CP phase from NO $v$ A, T2K and ICAL: a chronological progression," Nuclear Physics B, vol. 884, no. 1, pp. 274-304, 2014.

[10] I. Girardi, S. T. Petcov, and A. V. Titov, "Predictions for the leptonic Dirac CP violation phase: a systematic phenomenological analysis," The European Physical Journal C, vol. 75, no. 7, article 345, pp. 1-24, 2015.

[11] S. K. Kang and M. Tanimoto, "Prediction of leptonic CP phase in $A_{4}$ symmetric model," Physical Review D, vol. 91, no. 7, Article ID 073010, 8 pages, 2015.

[12] R. Aaij, B. Adeva, M. Adinolfi et al., "Precision measurement of $C P$ violation in $B_{S}^{0} \rightarrow J / \Psi K^{+} K^{-}$decays," Physical Review Letters, vol. 114, no. 4, Article ID 041801, 9 pages, 2015.

[13] P. Huber, M. Lindner, T. Schwetz, and W. Winter, "First hint for CP violation in neutrino oscillations from upcoming superbeamand reactor experiments," Journal of High Energy Physics, vol. 2009, no. 11, article 044, 2009.

[14] K. Bora, D. Dutta, and P. Ghoshal, "Determining the octant of $\theta_{23}$ at LBNE in conjunction with reactor experiments," Modern Physics Letters A, vol. 30, no. 14, Article ID 1550066, 22 pages, 2015.

[15] M. C. Gonzalez-Garcia, M. Maltoni, and A. Y. Smirnov, "Measuring the deviation of the 2-3 lepton mixing from maximal with atmospheric neutrinos," Physical Review D, vol. 70, Article ID 093005, 2004.

[16] A. Chatterjee, P. Ghoshal, S. Goswami, and S. K. Raut, "Octant sensitivity for large $\theta_{13}$ in atmospheric and long-baseline neutrino experiments," Journal of High Energy Physics, vol. 2013, no. 6, article 010, 2013.

[17] S. Choubey and A. Ghosh, "Determining the octant of $\theta_{23}$ with PINGU, T2K, NO $v$ A and reactor data," Journal of High Energy Physics, vol. 2013, no. 11, article 166, 2013.

[18] D. Kaur, Md. Naimuddin, and S. Kumar, "The sensitivity of the ICAL detector at India-based Neutrino Observatory to neutrino oscillation parameters," The European Physical Journal C, vol. 75, no. 4, article 156, pp. 1-8, 2015.

[19] A. Chatterjee, P. Ghoshal, S. Goswami, and S. K. Raut, "Octant sensitivity for large $\theta_{13}$ in atmospheric and long-baseline neutrino experiments," Journal of High Energy Physics, vol. 2013, no. 6, article 10, pp. 1-35, 2013.

[20] M. Ghosh, P. Ghoshal, S. Goswami, N. Nath, and S. K. Raut, "New look at the degeneracies in the neutrino oscillation parameters, and their resolution by T2K, NO $v$ A and ICAL," Physical Review D-Particles, Fields, Gravitation and Cosmology, vol. 93, no. 1, Article ID 013013, 2016.

[21] C. Adams, D. Adams, T. Akiri et al., "The long-baseline neutrino experiment: exploring fundamental symmetries of the universe," https://arxiv.org/abs/1307.7335.

[22] T. Akiri, D. Allspach, M. Andrews et al., "The 2010 interim report of the long-baseline neutrino experiment collaboration physics working groups," https://arxiv.org/abs/1110.6249.

[23] P. Adamson, C. Ader, D. Ayres et al., "NOvA proposal to build a 30 kiloton off-axis detector to study neutrino oscillations in the fermilab numi beamline," https://arxiv.org/abs/hep-ex/0503053.
[24] K. Abe, N. Abgrall, Y. Ajima et al., "Indication of electron neutrino appearance from an accelerator-produced off-axis muon neutrino beam," Physical Review Letters, vol. 107, no. 4, Article ID 041801, 2011.

[25] P. Adamson, D. J. Auty, D. S. Ayres et al., "Improved search for muon-neutrino to electron-neutrino oscillations in MINOS," Physical Review Letters, vol. 107, no. 18, Article ID 181802, 6 pages, 2011.

[26] D. Autiero, J. Aysto, A. Badertscher et al., "Large underground, liquid based detectors for astro-particle physics in Europe: scientific case and prospects," Journal of Cosmology and Astroparticle Physics, vol. 2007, no. 11, article 011, 2007.

[27] G. C. Branco, R. G. Felipe, and F. R. Joaquim, "Leptonic CP violation," Reviews of Modern Physics, vol. 84, no. 2, pp. 515-565, 2012.

[28] B. Pontecorvo, "Mesonium and antimesonium," Soviet Physics: Journal of Experimental and Theoretical Physics, vol. 6, p. 429, 1957.

[29] B. Pontecorvo, "Neutrino experiments and the problem of conservation of leptonic charge," Journal of Experimental and Theoretical Physics, vol. 26, no. 5, pp. 984-988, 1968.

[30] Z. Maki, M. Nakagawa, and S. Sakata, "Remarks on the Unified Model of Elementary Particles," Progress of Theoretical Physics, vol. 28, no. 5, pp. 870-880, 1962.

[31] F. Capozzi, G. L. Fogli, E. Lisi, A. Marrone, D. Montanino, and A. Palazzo, "Status of three-neutrino oscillation parameters, circa 2013," Physical Review D, vol. 89, Article ID 093018, 2014.

[32] G. L. Fogli, E. Lisi, A. Marrone, D. Montanino, A. Palazzo, and A. M. Rotunno, "Global analysis of neutrino masses, mixings, and phases: entering the era of leptonic CP violation searches," Physical Review D, vol. 86, no. 1, Article ID 013012, 2012.

[33] D. V. Forero, M. Tórtola, and J. W. F. Valle, "Neutrino oscillations refitted," Physical Review D, vol. 90, 2014.

[34] M. C. Gonzalez-Garcia, M. Maltoni, and T. Schwetz, "Updated fit to three neutrino mixing: status of leptonic CP violation," Journal of High Energy Physics, vol. 2014, article 52, 2014.

[35] V. Barger, D. Marfatia, and K. Whisnant, "Off-axis beams and detector clusters: resolving neutrino parameter degeneracies," Physical Review D, vol. 66, no. 5, Article ID 053007, 13 pages, 2002.

[36] H. Minakata and H. Nunokawa, "Exploring neutrino mixing with low energy superbeams," Journal of High Energy Physics, vol. 2001, article 10, 2001.

[37] A. S. Joshipura and K. M. Patel, "Fermion masses in $S O(10)$ models," Physical Review D, vol. 83, no. 9, Article ID 095002, 17 pages, 2011.

[38] M. Cebola, D. E. Costa, and R. G. Felipe, "Neutrino observables from predictive flavour patterns," The European Physical Journal C, vol. 76, article 156, 2016.

[39] K. A. Olive, K. Agashe, C. Amsler et al., "Review of particle physics," Chinese Physics C, vol. 38, no. 9, Article ID 090001, 2014.

[40] D. V. Forero, M. Tórtol, and J. W. F. Valle, "Neutrino oscillations refitted," Physical Review D, vol. 90, no. 9, Article ID 093006, 10 pages, 2014.

[41] G. L. Fogli, E. Lisi, A. Marrone, D. Montanino, A. Palazzo, and A. M. Rotunno, "Global analysis of neutrino masses, mixings, and phases: entering the era of leptonic CP violation searches," Physical Review D, vol. 86, no. 1, Article ID 013012, 2012.

[42] M. C. Gonzalez-Garcia, M. Maltoni, J. Salvado, and T. Schwetz, "Global fit to three neutrino mixing: critical look at present precision," Journal of High Energy Physics, vol. 2012, article 123, 2012. 
[43] K. Abe, J. Adam, H. Aihara et al., "Measurements of neutrino oscillation in appearance and disappearance channels by the T2K experiment with $6.6 \times 10^{20}$ protons on target," Physical Review D, vol. 91, no. 7, Article ID 072010, 50 pages, 2015.

[44] J. Bian, "First results of e appearance analysis and electron neutrino identification at NOvA," in Proceedings of the Meeting of the APS Division of Particles and Fields (DPF '15), Ann Arbor, Mich, USA, August 2015.

[45] https://indico.cern.ch/event/361123/contributions/856187/attachments/1136004/1625868/SK_atmospheric_kachulis_dpf2015.pdf.

[46] W. Buchmüller, P. Di Bari, and M. Plümacher, "Cosmic microwave background, matter-antimatter asymmetry and neutrino masses," Nuclear Physics B, vol. 643, no. 1-3, pp. 367390, 2002, Erratum in: Nuclear Physics B, vol. 793, no. 1-2, p. 362, 2008.

[47] R. N. Mohapatra and H.-B. Yu, "Connecting leptogenesis to CP violation in neutrino mixings in a tri-bimaximal mixing model," Physics Letters B, vol. 644, no. 5-6, pp. 346-351, 2007.

[48] P. S. Bhupal Dev, “TeV-scale leptogenesis," in XXI DAE-BRNS High Energy Physics Symposium, B. Bhuyan, Ed., vol. 174 of Springer Proceedings in Physics, pp. 245-253, 2016.

[49] P. S. B. Dev, C.-H. Lee, and R. N. Mohapatra, "TeV scale lepton number violation and baryogenesis," Journal of Physics: Conference Series, vol. 631, no. 1, Article ID 012007, 2015.

[50] G. Ghosh and K. Bora, "Resolving entanglement of CPV phase with octant of $\theta_{23}$, and leptogenesis," in Springer Proceedings in Physics, vol. 174, pp. 287-291, 2015.

[51] Z. Maki, M. Nakagawa, and S. Sakata, "Remarks on the unified model of elementary particles," Progress of Theoretical Physics, vol. 28, no. 5, pp. 870-880, 1962.

[52] N. Sahu and S. U. Sankar, "Bounds on neutrino masses from leptogenesis in type-II seesaw models," Physical Review D, vol. 71, no. 1, Article ID 013006, 5 pages, 2005.

[53] W. Rodejohann, "Non-unitary lepton mixing matrix, leptogenesis and low-energy CP violation," EPL (Europhysics Letters), vol. 88, no. 5, Article ID 51001, 2009.

[54] R. Kalita and D. Borah, "Constraining a type I seesaw model with $\mathrm{A}_{4}$ flavor symmetry from neutrino data and leptogenesis," Physical Review D, vol. 92, no. 5, Article ID 055012, 2015.

[55] R. Kalita and D. Borah, "Discriminating Majorana neutrino phases in the light of lepton flavor violation and leptogenesis in type I+II seesaw models," International Journal of Modern Physics A, vol. 30, no. 22, Article ID 1550130, 24 pages, 2015.

[56] E. I. Lashin, N. Chamoun, C. Hamzaoui, and S. Nasri, "Neutrino mixing and leptogenesis in $\mu-\tau$ symmetry," Physical Review D, vol. 91, no. 11, Article ID 113014, 25 pages, 2015.

[57] W.-C. Huang, "The dark side of $\theta_{13}, \delta_{C P}$, leptogenesis and inflation in type-I seesaw," Journal of High Energy Physics, vol. 2014, no. 11, article 083, 2014.

[58] M. Borah, D. Borah, and M. K. Das, "Discriminating Majorana neutrino textures in light of the baryon asymmetry," Physical Review D, vol. 91, no. 11, Article ID 113008, 2015.

[59] P. Di Bari and M. Re Fiorentin, "Supersymmetric SO(10)inspired leptogenesis and a new $\mathrm{N}_{2}$-dominated scenario," Journal of Cosmology and Astroparticle Physics, no. 3, Article ID 039, 2016.

[60] C. S. Fong, D. Meloni, A. Meroni, and E. Nardi, "Leptogenesis in SO(10)," Journal of High Energy Physics, vol. 2015, no. 1, article 111, pp. 1-21, 2015.

[61] G. Altarelli and D. Meloni, "A non supersymmetric SO(10) grand unified model for all the physics below $M_{G U T}$," Journal of High Energy Physics, vol. 2013, no. 8, article 21, 2013.
[62] P. Di Bari and L. Marzola, "SO(10)-inspired solution to the problem of the initial conditions in leptogenesis," Nuclear Physics B, vol. 877, no. 3, pp. 719-751, 2013.

[63] X.-D. Ji, Y.-C. Li, R. N. Mohapatra, S. Nasri, and Y. Zhang, "Leptogenesis in realistic SO(10) models," Physics Letters B, vol. 651, no. 2-3, pp. 195-207, 2007.

[64] S. Blanchet, P. S. Bhupal Dev, and R. N. Mohapatra, "Leptogenesis with TeV-scale inverse seesaw model in SO(10)," Physical Review D, vol. 82, no. 11, Article ID 115025, 11 pages, 2010.

[65] F. Buccella, D. Falcone, and F. Tramontano, "Baryogenesis via leptogenesis in SO(10) models," Physics Letters B, vol. 524, no. 3-4, pp. 241-244, 2002.

[66] R. N. Mohapatra, S. Nasri, and H.-B. Yu, "Leptogenesis, $\mu-\tau$ symmetry and $\theta_{13}$," Physics Letters $B$, vol. 615, no. 3-4, pp. 231239, 2005.

[67] J. A. Casas and A. Ibarra, "Oscillating neutrinos and $\mu \rightarrow e, \gamma$," Nuclear Physics B, vol. 618, no. 1-2, pp. 171-204, 2001.

[68] N. G. Deshpande and E. Keith, "Predictive fermion mass matrix Ansätze in nonsupersymmetric $\mathrm{SO}(10)$ grand unification," Physical Review D, vol. 50, no. 5, pp. 3513-3528, 1994.

[69] D.-G. Lee, R. N. Mohapatra, M. K. Parida, and M. Rani, "Predictions for the proton lifetime in minimal nonsupersymmetric SO(10) models: an update," Physical Review D, vol. 51, no. 1, pp. 229-235, 1995.

[70] F. Buccella, G. Miele, L. Rosa, P. Santorelli, and T. Tuzi, "An upper limit for the proton lifetime in $\mathrm{SO}(10)$," Physics Letters B, vol. 233, no. 1-2, pp. 178-182, 1989.

[71] A. Samanta, "The quark-lepton unification: LHC data and neutrino masses," Physics Letters B, vol. 728, pp. 11-14, 2014.

[72] S. Davidson and A. Ibarra, "A lower bound on the right-handed neutrino mass from leptogenesis," Physics Letters B, vol. 535, no. 1-4, pp. 25-32, 2002.

[73] K. Bora and G. Ghosh, "Neutrino masses and mixings using updated values of running quark and lepton masses," Journal of Physics: Conference Series, vol. 481, Article ID 012016, 2014.

[74] K. Bora, D. Dutta, and P. Ghoshal, "Probing sterile neutrino parameters with Double Chooz, Daya Bay and RENO," Journal of High Energy Physics, vol. 2012, no. 12, article 25, 2012.

[75] K. Bora and G. Ghosh, "Charged lepton flavor violation $\mu \rightarrow e \gamma$ in $\mu-\tau$ symmetric SUSY SO(10) mSUGRA, NUHM, NUGM, and NUSM theories and LHC," The European Physical Journal C, vol. 75, no. 9, article 428, 2015.

[76] G. t. Hooft, "Symmetry breaking through Bell-Jackiw anomalies," Physical Review Letters, vol. 37, no. 1, pp. 8-11, 1976.

[77] R. N. Mohapatra and X. Zhang, "Electroweak baryogenesis in left-right-symmetric models," Physical Review D, vol. 46, no. 12, pp. 5331-5336, 1992.

[78] F. R. Klinkhamer and N. S. Manton, "A saddle-point solution in the Weinberg-Salam theory," Physical Review D, vol. 30, no. 10, pp. 2212-2220, 1984.

[79] V. A. Kuzmin, V. A. Rubakov, and M. E. Shaposhikov, "On anomalous electroweak baryon-number non-conservation in the early universe," Physics Letters B, vol. 155, no. 1-2, pp. 36-42, 1985.

[80] S. Y. Khlebnikov and M. E. Shaposhnikov, "The statistical theory of anomalous fermion number non-conservation," Nuclear Physics B, vol. 308, no. 4, pp. 885-912, 1988.

[81] B. D. Fields, P. Molarto, and S. Sarkar, "Big bang nucleosynthesis," in Review of PDG-2014, Astrophysical Constants and Parameters, 2014.

[82] M. Malek, "Recent results from T2K," in Proceedings of the 17th Lomonoscov Conference on Elementary Particle Physics, Moscow State University, Moscow, Russia, August 2015. 

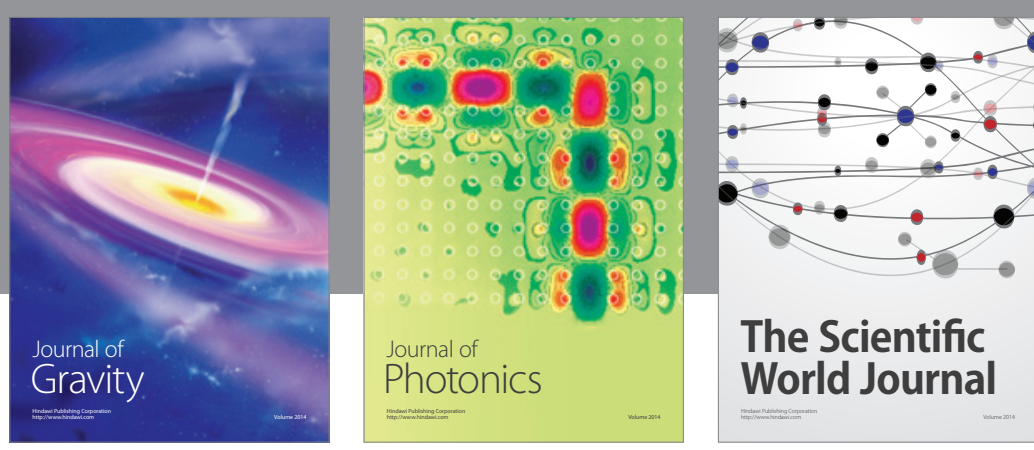

The Scientific World Journal
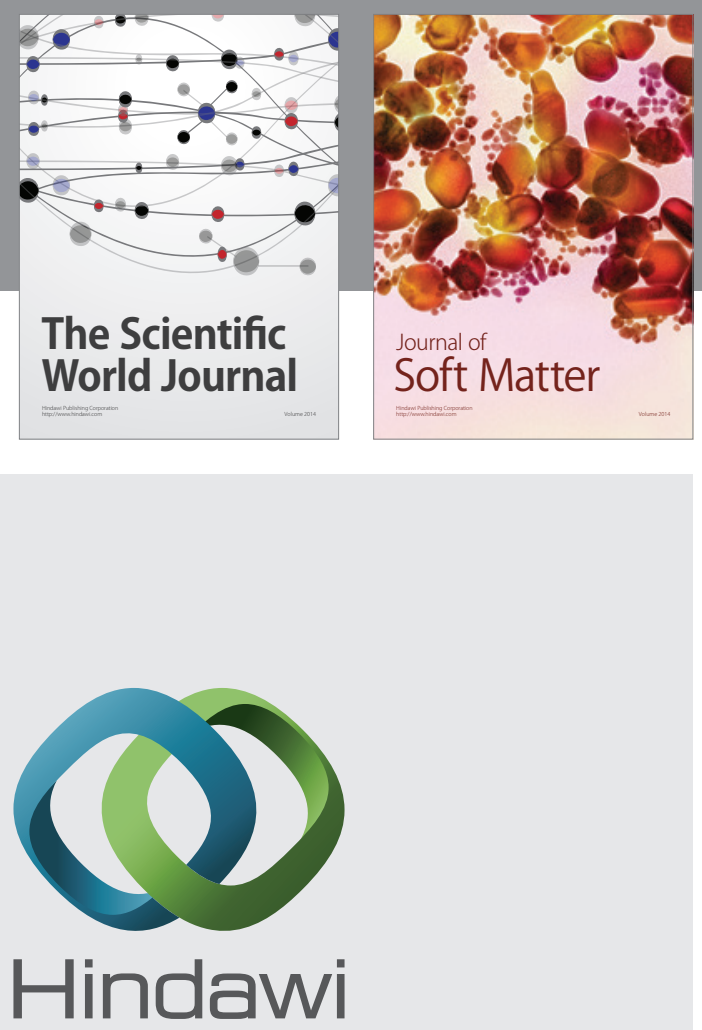

Submit your manuscripts at

http://www.hindawi.com

nternational Journal of

Statistical Mechanics
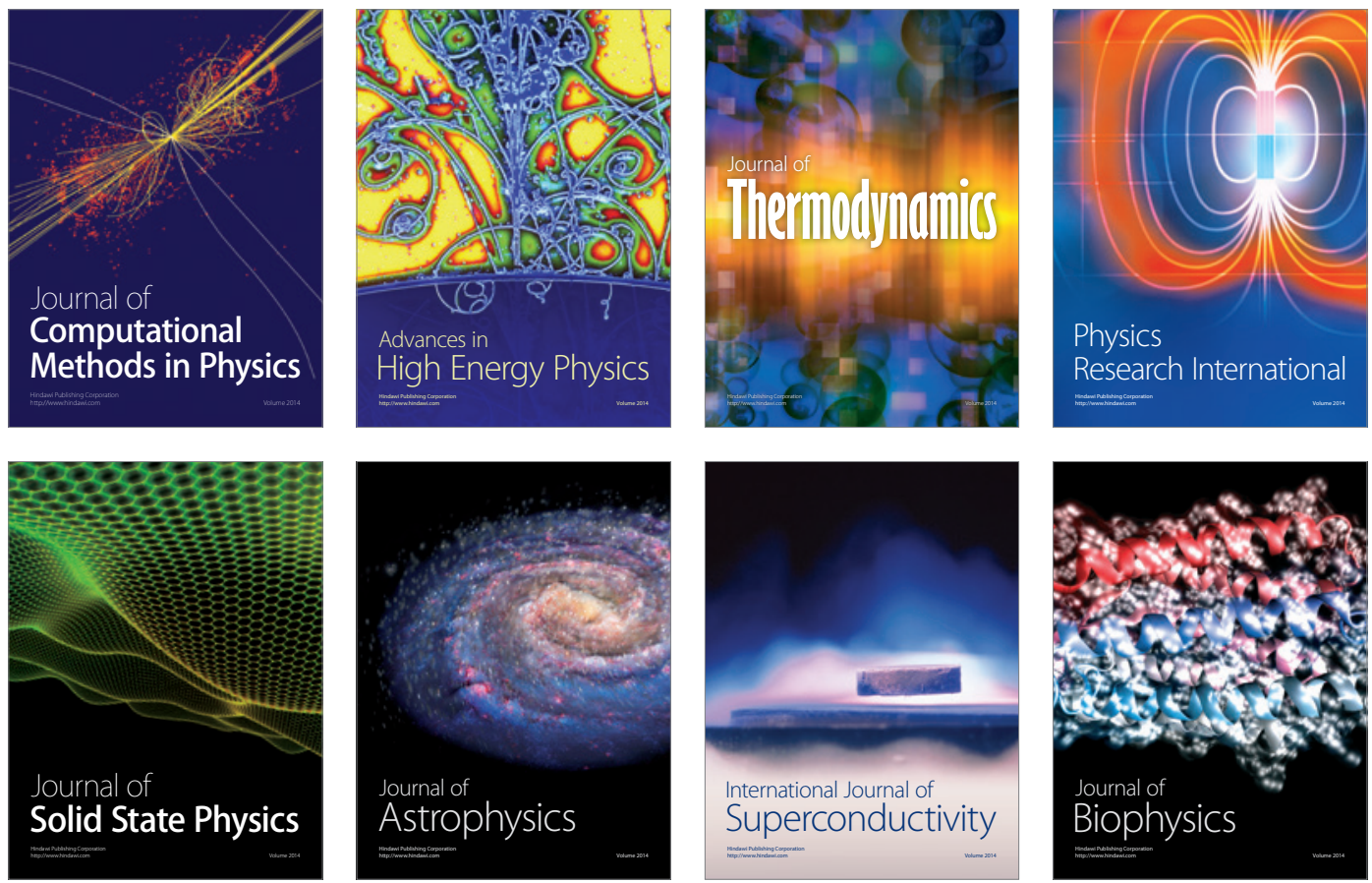
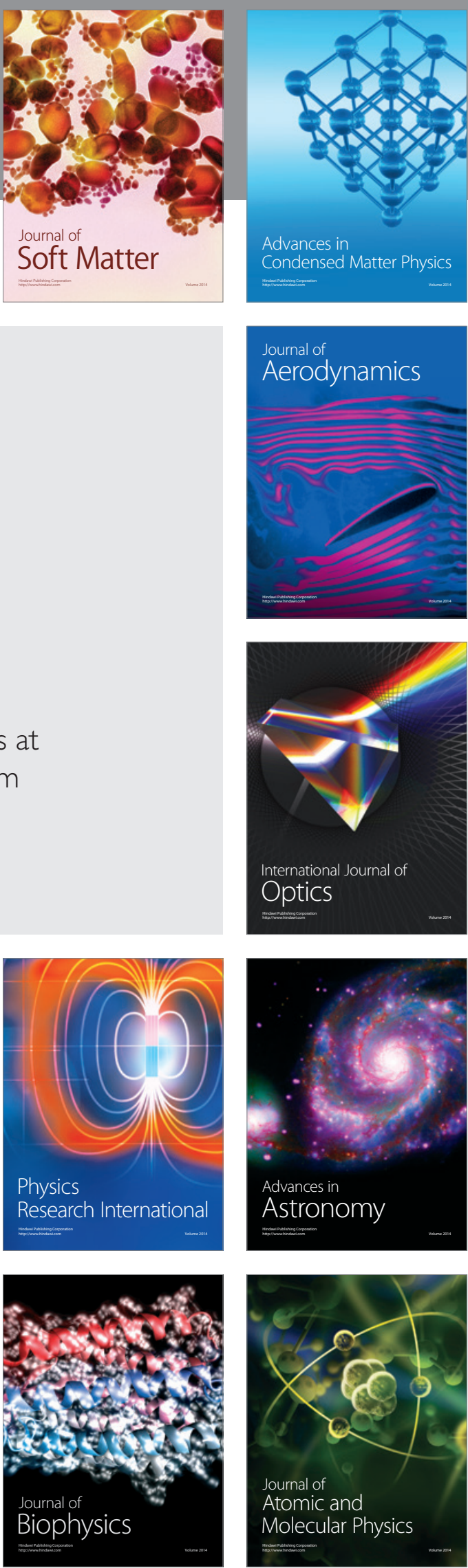